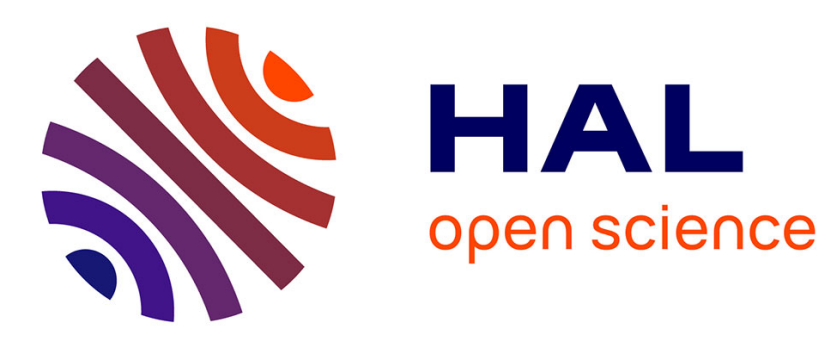

\title{
Hodology of the superior longitudinal system of the human brain: a historical perspective, the current controversies, and a proposal
}

Laura Vavassori, Silvio Sarubbo, Laurent Petit

\section{To cite this version:}

Laura Vavassori, Silvio Sarubbo, Laurent Petit. Hodology of the superior longitudinal system of the human brain: a historical perspective, the current controversies, and a proposal. Brain Structure and Function, 2021, 226 (5), pp.1363-1384. 10.1007/s00429-021-02265-0 . hal-03224904

\author{
HAL Id: hal-03224904 \\ https://hal.science/hal-03224904
}

Submitted on 12 May 2021

HAL is a multi-disciplinary open access archive for the deposit and dissemination of scientific research documents, whether they are published or not. The documents may come from teaching and research institutions in France or abroad, or from public or private research centers.
L'archive ouverte pluridisciplinaire HAL, est destinée au dépôt et à la diffusion de documents scientifiques de niveau recherche, publiés ou non, émanant des établissements d'enseignement et de recherche français ou étrangers, des laboratoires publics ou privés. 


\title{
Hodology of the superior longitudinal system of the human brain: a historical perspective, the current controversies, and a proposal.
}

\author{
Laura VAVASSORI ${ }^{1,2}$, Silvio SARUBBO ${ }^{3}$, Laurent PETIT ${ }^{1}$
}

${ }^{1}$ Groupe d'Imagerie Neurofonctionnelle, Univ. Bordeaux, CNRS, CEA, IMN, UMR 5293, F33000 Bordeaux, France.

${ }^{2}$ Center for Mind/Brain Sciences-CIMeC, University of Trento, Rovereto, Italy

${ }^{3}$ Division of Neurosurgery, Structural and Functional Connectivity Lab Project, Azienda Provinciale per i Servizi Sanitari (APSS), Trento, Italy

\section{Corresponding author:}

Laurent PETIT, Groupe d'Imagerie Neurofonctionnelle

Institut des Maladies Neurodégénératives CNRS UMR 5293

Université de Bordeaux

Centre Broca Nouvelle-Aquitaine - 3ème étage

146 rue Léo Saignat - CS 61292 - Case 28

33076 Bordeaux Cedex, France

Email: laurent.petit@u-bordeaux.fr 


\section{Highlights}

- A fundamental issue in human brain structural connectivity is the lack of a consensus upon the definition of white matter pathways.

- The Superior Longitudinal System (SLS), encompassing the arcuate (AF) and the superior longitudinal fasciculi (SLF), is an illustrative example of this issue.

- We propose to conceive the SLS organization as an orderly and continuous wiring diagram rather than in terms of individualized fascicles. 


\begin{abstract}
The description of human white matter pathways experienced a tremendous improvement thanks to the advancement of neuroimaging and dissection techniques. The downside of this progress is the production of redundant and conflicting literature, bound by specific studies' methods and aims. The Superior Longitudinal System (SLS), encompassing the arcuate (AF) and the superior longitudinal fasciculi (SLF), becomes an illustrative example of this fundamental issue, being one of the most studied white matter association pathways of the brain. Herein we provide a complete illustration of this white matter fiber system's current definition, from its early descriptions in the $19^{\text {th }}$ century to its most recent characterizations. We propose a review of both in vivo diffusion magnetic resonance imaging-based tractography and anatomical dissection studies, enclosing all the information available up to date. Based on these findings, we reconstruct the wiring diagram of the SLS, highlighting a substantial variability in the description of its cortical sites of termination and the taxonomy and partonomy that characterize the system. We aim to level up discrepancies in the literature by proposing a parallel across the various nomenclature. Consistently with the topographical arrangement already documented for commissural and projection pathways, we suggest approaching the SLS organization as an orderly and continuous wiring diagram, respecting a medio-lateral palisading topography between the different frontal, parietal, occipital, and temporal gyri rather than in terms of individualized fascicles. A better and complete description of the fine organization of white matter association pathways' connectivity is fundamental for a better understanding of brain function and their clinical and neurosurgical applications.
\end{abstract}

\title{
Keywords
}

Human white matter anatomy, Dissection, Tractography, Association pathways, Arcuate fascicle, Superior longitudinal fascicle 


\section{Declarations}

Funding: No particular funding.

Conflicts of interest/Competing interests: No conflict of interest for any of the three authors.

\section{Ethics approval:}

- The present manuscript is not submitted elsewhere for simultaneous consideration;

- The submitted work is original and has not been published elsewhere in any form or language (partially or in full);

- No re-use of material to avoid the concerns about text-recycling ('self-plagiarism') in the present manuscript;

- In the context of the present review article, data, by definition already published, are presented clearly, honestly, and without fabrication, falsification, or inappropriate data manipulation (including image-based manipulation);

- No data, text, or theories by others are presented as if they were the authors' own ('plagiarism'). Proper acknowledgments to other works have been given (this includes material that is closely copied (near verbatim), summarized, and/or paraphrased), quotation marks (to indicate words taken from another source) are used for verbatim copying of material, and permissions secured for copyrighted material.

Consent to participate (include appropriate statements): not applicable for a review article

Consent for publication (include appropriate statements): not applicable for a review article

Availability of data and material (data transparency): not applicable for a review article

Code availability (software application or custom code): no specific code developed, not applicable. 
"As chroniclers of an incremental process, [historians of science] discover that additional research makes it harder, not easier, to answer questions like: When was oxygen discovered? Who first conceived of energy conservation? Increasingly, a few of them suspect that these are simply the wrong sorts of questions to ask. Perhaps science does not develop by the accumulation of individual discoveries and inventions. [...] Men whose research is based on shared paradigms are committed to the same rules and standards for scientific practice. That commitment and the apparent consensus it produces are prerequisites for normal science, i.e., for the genesis and continuation of a particular research tradition." (Kuhn, 1996)

\section{Introduction}

The hodology, from the ancient Greek hodos, refers to a road or pathway and aims to describe the origins, course, and site of termination of white matter (WM) fiber pathways and the study of the structure and composition of these pathways (Nieuwenhuys, 1998). During the past 15 years, the description of human WM pathways experienced a tremendous improvement thanks to the advancement of neuroimaging and dissection techniques. Still, their general hodology has yet to be developed. A fundamental issue in human brain structural connectivity is the lack of a consensus upon the definition of WM pathways. Going back to the literature reveals a quite confusing background for their anatomical definition and taxonomy (Swanson, 2015). Since the time of the gross dissections carried out in the 17th century by Willis, Steno, Malpighi, Vicq d'Azyr, and others, everyone has agreed for centuries that the organization of the cerebral WM is complex. The continuous improvement of ex-vivo investigation techniques, starting from brains' fixation in alcohol at the turn of the $19^{\text {th }}$ century to more recent adaptations of Klingler dissection approach (Klingler, 1935), e.g., cortex-sparing fiber dissection and photogrammetry (De Benedictis et al., 2018; Martino et al., 2011), still produces innovated knowledge in the field.

Moreover, the advent of diffusion magnetic resonance imaging (dMRI) (for a detailed review, see Leemans (2019) and related articles) introduced the possibility to study the structural connectivity in-vivo. The outcome is a vast amount of updated knowledge that enlarges our understanding of the human brain connectome. However, the use of different 
approaches with different resolutions and different paradigms has led to contrasting results. It becomes remarkedly true for the long WM association pathways, namely bundles of fibers that connect different cortical areas within the same hemisphere. The main hazard in describing these pathways consists of the artifacts that both in-vivo and ex-vivo techniques can produce, mainly when dealing with crossing fibers and cortical terminations. It is related to the nature of the brain's WM, made of axons densely packed into the crossing, kissing, and bending fibers' bundles. Such an arrangement can led to uncertainty when anatomically tracking a pathway to its terminations and can 'trick' an algorithm into tracking a nearby bundle's diffusivity direction. To date, cortex-sparing Klingler dissection (Martino et al., 2011) and dMRI-based tractography algorithms (Jeurissen et al., 2019) are suitable alternatives for studying the structural connectivity of the human brain at the macroscopic level.

The technical limitations mentioned above also faced a more general issue regarding the human brain neuroanatomy, namely the lack of anatomical ground truth that has led to several taxonomic definitions of the WM association bundles (Mandonnet et al., 2018). As the field has advanced, the attribution of new features to previously described tracts has modified their categorization and their subcomponents' description. Moreover, various nomenclatures have been used while referring to the same tract, depending on the study's aim. Different rules have been used to conceptualize knowledge: embracing functional or anatomical criteria has shaped the definition of bundles of fibers in diverse ways. It resulted in conflicting literature (Mandonnet et al., 2018; Panesar and Fernandez-Miranda, 2019), an unsteady base for scientific progress. The adhesion to a common taxonomy based on anatomical criteria could reduce redundancy in the literature and represent a gain in understanding and sharing knowledge.

Focusing on the superior longitudinal system (SLS), this review aims to enlighten the more prominent contradictions in the literature (Mandonnet et al., 2018) and advance the possibility of describing the brain's structural connectome with the sole adoption of anatomical priors. Indeed, the presence of lateral ventricles and subcortical grey nuclei constrains the course of longitudinal association fibers leaving the frontal lobe to follow a path either superiorly, above the superior limiting sulcus of the insula, or inferiorly, along with the external capsule (Mandonnet et al., 2018). We consider part of the SLS all association fibers leaving the frontal lobe and coursing posteriorly, above the superior limiting sulcus of the insula, to the parietal, temporal, and occipital cortices. Consequently, SLS encompasses those WM pathways classically described in the literature as the superior longitudinal fasciculus (SLF) and/or the arcuate fasciculus (AF). 
To stress the importance of a consensus, we reviewed the studies that contributed to SLF and AF's current definition. Our first goal is to understand what makes these two bundles similar or different throughout the history of their description. We considered their early reports, from Reil's and Burdach's seminal works to the development of Klingler's dissection technique and up to the advent of tractography. Therefore, we reviewed 46 peer-reviewed articles, including prominent studies that focus on the anatomical description of SLF and/or AF by using either Klingler microdissection or dMRI-based tractography or both. For completeness, we also report the main functional roles which have reliably been attributed to the AF and the SLF. Indeed, as the adoption of different nomenclatures of these structures and/or portions of, may bias the anatomical characterization of a WM tract, it also hinders the comparison of evidences in a functional framework.

Since the literature analysis does not reveal an agreement on the anatomical definition of these WM pathways, we propose a more general organization scheme for characterizing the SLS's long association fibers. We suggest considering them in light of the topographical arrangement already described for commissural and projection pathways, namely respecting a layer-based topography between the different frontal, parietal, occipital, and temporal gyri rather than in terms of individualized fascicles.

\section{The first descriptions of SLS in terms of SLF, AF, or both.}

As mentioned in the neuroanatomical terminology lexicon (Swanson, 2015), early macrodissection studies of the WM of the human brain at the turn of the 19th century proposed the existence of a prominent arched, longitudinally oriented system of long WM fibers which mainly interconnected the frontal region to parietal, occipital, and temporal cortices. Thus, SLS's current definition corresponds to the earliest descriptions of association pathways in the human brain. The very first description is attributed to Johann Christian Reil. He delineated what he called the intermediate white matter (in the original German, "intermediäre Marksubstanz") in an early publication (Reil, 1809), but illustrated in Plate XII in Reil (1812) (Figure 1A). These works have been translated into English a few years later by Herbert Mayo as follows: "Besides what is strictly the outer wall of the capsule, there may be raised, after the general removal of the convolutions, other medullary fasciculi, which have passed from the centre of the base of one convolution to that of another, connecting not merely adjoining, but even remote convolutions: these are especially found within the convolutions of the roof of the fissura Sylvii, whence they pass round the island to convolutions in the middle lobe (authors 
note, i.e., temporal lobe): this structure is probably general and is probably intended, with the hamular fasciculi (authors note, i.e., uncinate fascicle), for the common purpose of associating together in action the convolutions of the great divisions of either hemisphere" (Mayo, 1823). After this first detailed characterization of long-range fascicular connection in the human brain, Friedrich Burdach was the first to characterize a series of association fiber bundles introducing the term of arcuate fasciculus as translated from his second book and referring himself from Reil work: "The arcuate fascicle (Bogenbündel) is a thick layer of matter which radiates on the outer surface of each hemisphere in the same orientation as the cingulum (Zwinge), but on the lateral surface, from the temporal lobe (Unterlappen) backwards and upwards into the parietal lobe (Hinterlapen), and encircling the Sylvius fissure (Unterspalte), through the frontal lobe (Oberlappen)." (Burdach, 1822). Burdach referred to the arcuate fascicle in several of the illustrations that accompanied his work. Figure 1B illustrates the one mainly dedicated to the description of the AF.

Macrodissection studies continued throughout the $19^{\text {th }}$ century with magnificent illustrations alternatively depicting AF and SLF without any clear distinction between them, without even naming them but only representing them (Figure $1 \mathrm{C}-\mathrm{E}$ ).

The turn of the 20th century saw a series of essential works describing such association pathways as a single entity. Jules Dejerine and Augusta Dejerine-Klumpke (Dejerine and Dejerine-Klumpke, 1901, 1895) indiscriminately named this ensemble the arcuate fascicle of Burdach or superior longitudinal fascicle ("faisceau arqué de Burdach ou faisceau longitudinal supérieur”). We have chosen to reproduce the whole definition given by the Dejerines because it is by far the complete definition ever proposed, although paradoxically only illustrated with semi-schematic figures (Figure 2). The authors use both terms simultaneously when describing this structure with unprecedented precision as translated from the first book (pg. 756-757): "The arcuate fascicle is to the external surface of the hemisphere what the cingulum is to the internal surface; situated at the base of the convolutions of the Sylvian operculum, outside the fibres of the foot of the corona radiata and at the level of the trunk of the corpus callosum, this bundle describes, like the cingulum and the uncinate fascicle, a curve open at the bottom and in front. In dichromate or alcohol-hardened brain dissections, the arcuate fascicle only appears as a compact bundle in the parietal region of the operculum; parallel to the upper edge of the putamen, its lowest and most superficial fibres reach the upper marginal sulcus of the insula, dissociate the upper edge of the claustrum (Avant-mur), cover outside the fibres of the foot of the corona radiata, and contribute to forming the upper part of the external capsule. At the posterior border of the Sylvius fissure, the arcuate fascicle 
describes a curve with an anterior concavity, which embraces the posterior edge of the putamen, then its fibres fan out, intertwine with the fibres of the corona radiata and the corpus callosum, and run along the base of the parieto-occipito-temporal convolutions. The most superficial fibres are carried forward, externally covering the uncinate fascicle, and radiate into the crest of the anterior part of the superior temporal gyrus (T1); the following ones end in the posterior segment of the superior temporal gyrus and in the middle temporal gyrus (T2), where they intersect with the fibres of the corona radiata and the corpus callosum. The deepest ones, finally, radiate into the crests of the supramarginal gyrus, the angular gyrus (pli courbe), and the gyri of the external face of the occipital lobe $(\mathrm{O} 1, \mathrm{O} 2, \mathrm{O} 3)$. As one moves away from the insula's posterior marginal sulcus, these fibres become increasingly difficult to follow due to their entanglement with the corpus callosum and projection fibres. In the vertico-transverse sections of brains hardened in alkaline dichromate, the cross-sectional area of the arcuate fascicle is triangular; its lower-inner angle corresponds to the external capsule and is inserted between the latter and the claustrum (Avant-mur), its lower-external angle corresponds to the base of the Sylvian operculum convolutions, and its upper angle is lost in the layer of surrounding fibres. Poorly delimited on the outside and at the bottom, where it merges imperceptibly with the white mass of the convolutions of the Sylvan operculum, the arcuate fascicle is very well delimited medially, where it stands at the foot of the corona radiata: its fibres are in fact perpendicular to the direction of the radiating fibres. At the level of the supramarginal gyrus, the arcuate fascicle curves downwards and forwards to reach the temporal lobe; it merges backward with the layer of vertical fibres of the convexity of the occipital and parieto-temporal regions, a layer constituted by Wernicke's vertical occipital fascicle."

Thus, the Dejerines made no distinction between the arcuate fasciculus and the superior longitudinal fasciculus; the ensemble was defined as a single bundle interchangeably named along with the text and figure captions of their books. Dejerines' first studies were unable to follow the fibers to their frontal termination. They have reported the difficulty in following these fibers as they merge with callosal and projection fibers at the level of the circular sulcus of the insula. This description was then modified considering von Monakow's observation of the tract in 1897 (von Monakow, 1897), with the attribution of rostral endings to the inferior frontal gyrus (Dejerine and Dejerine-Klumpke (1901); see Figure 2 in Dick and Tremblay (2012) for this historical point of view).

A crucial step was taken in the early 1930s to understand the human WM tracts' organization with a new method of dissection developed by Josef Klingler (Agrawal et al., 2011). In his 
famed Atlas Cerebri Humani with the collaboration of Eugen Ludwig (Ludwig and Klingler, 1956), only the superior frontal fascicle is mentioned and encompassed the arcuate fascicle (Figure 3).

The first 150 years of the description of the SLF/AF organization shaped researchers' approach up to the last decade (Bernal and Altman, 2010), highlighting the importance of having a shared terminology, which reflects the most recent discoveries in the field, or rather, the actual anatomy. Türe et al. (2000)'s dissection study proposed the nomenclature 'superior longitudinal (arcuate) fasciculus' to describe a bundle of fibers connecting perisylvian frontal, parietal and temporal cortices. Catani et al. (2002) adopted the same definition when describing three-dimensional structures in the human brain with virtual in-vivo interactive dissection (VIVID) based on the emerging diffusion MRI technique. The labels superior longitudinal fasciculus and arcuate fasciculus were thus merged to form a single term, a legitimate choice reflecting the complete overlapping of the underlying concepts. The propensity to consider these anatomical structures as the same and only one is also reflected in some works which referred to all perisylvian fibers as superior longitudinal fasciculus or arcuate fasciculus alternatively. Mori et al. (2002) adopted the definition superior longitudinal fasciculus, while Lawes et al. (2008) referred to all perisylvian tracts as arcuate fasciculus. This terminology started to be questioned by introducing an inner subdivision into the tract's subcomponents (Catani et al., 2005). However, some more recent works seem to be still influenced by this concept. For example, Bernal and Altman (2010) explicitly refer to the differentiation between SLF and AF, but when defining the rostral termination of SLF state "The main anterior connection terminal of the SLF (and consequently of the AF) [...]", suggesting a certain degree of identification between AF and SLF. A reasonably straightforward summary has been proposed by Dick and Tremblay (2012): "Historically, the superior longitudinal fasciculus and arcuate fasciculus have been viewed as synonymous, non-dissociable fiber pathways connecting the inferior frontal gyrus with the inferior parietal lobule and temporal lobe. It is only recently that there have been some attempts to dissociate parts of the SLF/AF, and generally these promote the notion that the arcuate fasciculus represents a partition of a broader superior longitudinal fasciculus."

\section{How have AF and SLF been recently described?}

Thanks to its non-invasiveness, the advent of diffusion MRI enabled many studies on human WM, leading to the disclosure of a more complex SLS organization (Catani et al., 2005; 
Makris et al., 2005). The previous paradigm was questioned, with the endorsement of a new categorization. Based on the novel evidence available, fibers connecting the frontal lobe with the parietal lobe were attributed to the SLF, while those reaching the temporal cortex were ascribed to the AF (Kamali et al., 2014; Sarubbo et al., 2016; Thiebaut de Schotten et al., 2012). Still, the interrelation between the two bundles has been debated. Some authors classify AF as a subcomponent of SLF, suggesting a hierarchy within the bundle. For instance, FernándezMiranda et al. (2008) refer to frontotemporal tracts extracted via diffusion tensor imaging (DTI) and anatomical dissection as the arcuate component of the superior longitudinal fasciculus. More recent neuroimaging studies (Conner et al., 2018; Kamali et al., 2014), as well as ex-vivo Klingler's dissection studies (De Benedictis et al., 2014; Martino et al., 2013, 2011; Zemmoura et al., 2014), support this characterization of SLF-AF partonomy.

Another line of research considers SLF and AF as two separate entities. This categorization is hardened by different hemispheric lateralization patterns shown by the two bundles: SLF was found to be equally extended in the two hemispheres, while AF exhibits higher connectivity in the left hemisphere compared to the right (Vassal et al., 2016). Moreover, Thiebaut de Schotten et al. (2012) addressed the study of these connections' phylogenic development, stressing both SLF general preservation and AF significant modification through evolution. The differentiation of the two structures at the anatomical level was made possible by the improvement of both dissection (Monroy-Sosa et al., 2019) and diffusion imaging techniques (Rojkova et al., 2016; Vassal et al., 2016), but general support to this thesis also comes from the implementation of functional information to structural data. Intraoperative stimulation of WM and lesion studies revealed AF to be involved in language processing (phonological, lexical and semantic), while SLF mediates more spatial and motor skills, including spatial attention, higher aspects of motor function, and speech articulation (Güngör et al., 2017; Sarubbo et al., 2020a, 2015; Yagmurlu et al., 2016; Zacà et al., 2018). Altogether, these shreds of evidence stand for a disjoint categorization of the two bundles.

This section focuses on the main partitioning into sub-components proposed for both SLF and AF. Methodologically, we organized the information into different categories collecting all the nomenclatures advanced across the literature and reflecting the most persuasive proposals for the segmentation of both SLF and AF, i.e., the SLS. For each category, we provide a schematic representation of the components considered (Figure 4 to 8 ), collecting all their different attributed labels within a particular line of research. This information is complemented with each component's wiring diagram for each taxonomy: the overall connectivity pattern of a sub-tract is determined by the conjunction of all the descriptions 
endorsing that particular categorization. The different proposals coming from neuroimaging and dissection studies that contributed to the anatomical report of a tract are represented by a line connecting two regions of interest (ROIs) and referenced for each ROI (Figure 4 to 8). Within the wiring diagram, the overlap of more descriptions is represented by each line's width, which is proportional to the number of studies supporting a specific proposal. When addressing the tracts' various delineations in terms of terminations sites, we will present the findings by a "gyral/sulcal" nomenclature based on macroscopic anatomical landmarks. Indeed, the inherent confusion of the description of these bundles in the current literature comes in part from the mixed description of their cortical territories in terms of Brodmann areas (BAs) and/or large cortical regions and/or gyral/sulcal macroscopic terms. The variability of the cytoarchitectonic areas in the human brain does not allow a reliable description of brain anatomical features in terms of BAs (Rajkowska and Goldman-Rakic, 1995). The uncertainty about the boundaries of large regions (e.g., ventral premotor cortex, dorsolateral prefrontal cortex, etc.) makes the delineation of the terminal territories of the fascicles unclear in these terms.

On the other hand, the gyral/sulcal description can be applied to each individual where such landmarks can be easily identified in anatomical and diffusion MR images (e.g., Destrieux et al., 2017; Tzourio et al., 1997). The anatomical labeling used in the original studies will be mentioned for their first use in the text; we will add their equivalence in gyral/sulcal terms when necessary. To provide a schematic representation with the wiring diagrams, the definitions of the sites of termination specifying only the partial involvement of an ROI (e.g., anterior, posterior, etc.) are enlarged to the whole gyrus, exception made for the IFG, whose subdivision in pars opercularis, pars triangularis and pars orbitalis is justified by macroanatomical features. Further comparison both across and between standpoints is prompted via the use of the same color code.

\subsection{AF (Figure 4)}

Language models fundamentally drove the classical definition of the arcuate fasciculus: it represents Wernicke's postulation of a structural link between Broca's area for speech production and Wernicke's area for speech understanding. Therefore, its cortical terminations were attributed to posterior inferior frontal regions and caudal temporal regions, respectively. Its comprehensive course is influenced by the Sylvian fissure's presence, which determines its characteristic arched-form at the border with parietal and temporal lobes (Geschwind, 1967). The characterization of this direct connection between frontal and temporal lobes was further complemented thanks to both dissection and neuroimaging studies, which provided a more 
detailed description of the extension of its wiring (Figure 4). At the functional level, different studies using direct electrical stimulation (DES) during awake surgery or lesion models (in particular stroke) supported the importance of the AF in language processing, supporting phonological integration (Maldonado et al., 2011; Sarubbo et al., 2020a, 2015). Papagno et al. (2017) suggest that this WM pathway might be involved in verbal short-term memory, specifically conveying single-items information. Still, other studies propose that the AF could be relevant also for non-linguistic abilities. For instance, a lesion mapping study by Herbet et al. (2014) stresses that this structure in the right hemisphere plays a role in emotion recognition, for the identification of other's mental states. Moreover, Umarova et al. (2010), by combining DTI-based tractography and fMRI, described the involvement of the AF in the right hemisphere also in visuo-spatial attentional tasks, supporting the detection of changes in the environment.

Frey et al. (2008) extracted the AF with high angular resolution fiber tractography. They described its course from the posterior part of the superior temporal gyrus (pSTG) to the posterior dorsolateral frontal cortex (MFG). By using diffusion tensor imaging (DTI), Vassal et al. (2016) addressed the study of AF cortical terminations in both hemispheres. In the left hemisphere, streamlines connect the middle temporal gyrus (MTG) and STG to the ventral premotor cortex (vPrCG) and inferior frontal gyrus (IFG) - connections to pars opercularis (IFGop) are found consistently across subjects. In contrast, the extent to which the temporal lobe is connected with pars triangularis (IFGtri) is more variable. On the other hand, the right hemisphere shows uniform connectivity only between MTG and $\mathrm{VPCG}$; extensions towards STG and IFG were found in fewer participants. In a few subjects, the AF of both hemispheres also showed rostral connectivity with the middle frontal gyrus (MFG). Through blunt microdissection, Sarubbo et al. (2016) were able to describe the AF in its entire course. Posterior terminations were reported in STG, in the posterior and middle thirds of the MTG, and the middle third of inferior temporal gyrus (ITG). Anteriorly, AF fibers reach the anterior portion of the $\mathrm{vPrCG}$, IFGop, and the ventral portion of the posterior MFG. Other authors considered AF cortical sites of termination without partitioning the bundle, but the adopted terminology was shaped by how they conceptualized SLF-AF partonomy. For instance, two important studies compared DTI data with dissection studies to strengthen their definition of this pathway. Fernández-Miranda et al. (2008) tracked the so-called arcuate segment of the SLF to its cortical terminations in posterior temporal regions and prefrontal areas. Martino et al. (2011) defined the SLF temporofrontal segment as a connection running from posterior MTG and posterior ITG to the frontal operculum, which encompasses vPrCG and both IFGop and IFGtri. 


\subsubsection{AF tripartite subdivision (Figure 5)}

Catani et al. (2005) proposed the first internal subdivision of the AF, stressing that this connection would consist of three different components (Figure 5a). Fiber tracking based on DTI revealed two parallel pathways connecting the frontal to the temporal lobe: the direct pathway, also defined as the long segment, connects the ventrolateral frontal lobe (IFG) and the posterior part of the MTG and of the STG with no interruption; the indirect pathway involves the same cortical areas in frontal and temporal lobes, but it is composed by an anterior segment, connecting frontal and parietal cortices, and a posterior segment, which runs through the parietal and temporal lobes. The parietal region involved in the tract was identified as the inferior parietal cortex (BAs 39, 40, i.e., angular gyrus (AG) and supramarginal gyrus (SMG), respectively). Barrick et al. (2006)'s DTI study reproduced the existence of both long and posterior segments of the AF, providing a more detailed definition of their sites of termination. The long segment was found to connect the STG and the posterior part of the MTG and the ITG to the precentral gyrus (PrCG) and IFGop; the posterior segment originates from the same areas in the temporal lobe, but it terminates in both SMG and AG. Lawes et al. (2008) compared neuroimaging data with ex-vivo studies. They found the direct segment running from middle and posterior MTG to IFGop and vPrCG; the anterior segment connects the long segment's rostral areas with AG, while the posterior segment links posterior MTG with both AG and SMG. Moreover, the authors proposed further dividing the long and the anterior segments into two sub-tracts, depending on whether fibers end up in IFGop or vPrCG.

The tripartite subdivision of the AF was also supported by a subsequent diffusionweighted imaging (DWI) study (Rojkova et al., 2016). The long and the anterior segments were described: the direct pathway was reported to connect IFGop with the auditory cortex in the posterior STG, the posterior MTG, and the ITG, while the anterior segment arises in vPrCG and terminates in the postcentral gyrus (PoCG) and the SMG. A recent study by Forkel et al. (2020) investigated the anatomical correlates of repetition deficits in primary progressive aphasia, addressing the degree of AF fibers atrophy via DTI-based tractography. To this end, they assume the classical segmentation of the bundle into direct and indirect pathways, defining the long segment as a connection between Broca territory, including posterior IFG and PrCG, and Wernicke territory, grouping the middle part of STG, the posterior MTG, and the Sylvian temporo-parietal area. The indirect pathway would connect these regions to Geschwind's territory, represented by the anterior SMG, the AG, and the temporo-parietal junction, through the anterior and the posterior segments, which provide fronto-parietal and temporo-parietal connectivity, respectively. 
A particular matter of discussion concerned whether the anterior and posterior segments should be considered parts of the AF. This concern is reflected by some authors' choice of applying the tripartite categorization to a broader ensemble of WM fibers, namely to all perisylvian connections (Figure 5b). While addressing connectivity of the right hemisphere through DTI, Gharabaghi et al. (2009) defined the direct component, running from the superior temporal cortex (STG) to the lateral frontal cortex (MFG, IFG) as the classical arcuate fasciculus. The anterior segment, connecting inferior parietal (AG, SMG) and lateral frontal cortices (MFG, IFG), as well as the posterior segment, whose fibers link superior temporal (STG) and inferior parietal cortices (AG, SMG), are addressed as perisylvian connections. In this research line, Martino et al. (2013) described the tracts by combining DTI with cortexsparing dissection studies. The long segment, which is also referred to as the AF, was running from both ITG and MTG to the vPrCG, posterior IFG, and in some cases to MFG. For what concerns the other perisylvian tracts, the anterior segment connects the SMG and the posterior STG (few specimens showed connections to the AG) with vPrCG. Simultaneously, the posterior component connects posterior MTG with the AG (in some cases, connections were also found with the SMG and the ventral portion of the superior parietal gyrus (SPG)). Zemmoura et al. (2014) isolated fiber tracts via dissection and reconstructed them in an MRI reference space via the FIBRASCAN method. They described the long segment, or AF, as running between IFGop, IFGtri, and posterior MTG. The posterior segment was defined as a connection between the inferior parietal cortex and the posterior part of the STG and of the MTG. Interestingly, they proposed the anterior segment, connecting vPrCG and the IPL, to be a part of the SLF, specifically SLF III.

\subsubsection{AF additional subdivisions (Figure 6)}

In addition to the tripartite stance, other internal subdivisions of the AF were proposed. Despite the different labels attributed to the various segments, the following nomenclatures all point to the same type of categorization for the AF fibers, which have been subdivided into two major pathways depending on their origin sites in the temporal lobe (Figure 6). Through DWI, Thiebaut de Schotten et al. (2012) distinguished two subsets of fibers within the AF. They advanced the differentiation between a set of fibers connecting posterior STG with BAs 44 and 45 (IFGop and IFGtri, respectively) and a more massive subtract running from the MTG and ITG to the vPrCG and posterior regions of MFG and IFG. This proposal was first envisaged by Glasser and Rilling (2008)'s DTI study, which identified within the system of the AF an STG pathway, linking posterior STG to IFGop and $\mathrm{vPrCG}$, and an MTG pathway, encompassing 
streamlines connecting MTG and posterior ITG to IFGop, IFGtri, vPrCG, and the posterior MFG.

Different terminologies for categorizing AF fibers stem from integrating diffusion data and dissection studies within this research line. Fernández-Miranda et al. (2015) segmented human AF through diffusion spectrum imaging (DSI) and microdissection. They defined it to be composed by an inner or ventral pathway, connecting IFGop to STG and the anterior part of MTG, and by an outer or dorsal pathway, originating in $\mathrm{VPCG}$ and posterior MFG and ending up in posterior parts of MTG and ITG. This proposal was supported by Yagmurlu et al. (2016) via the combination of DTI and dissection studies. They re-defined the ventral pathway as a connection linking posterior and middle STG and middle MTG to IFGop, vPrCG, and, in some cases, to IFGtri. The temporal terminations of the dorsal pathway remained unvaried from Fernández-Miranda et al. (2015)'s proposal, while its anterior extremities were attributed to IFGop, vPrCG, and posterior MFG.

\subsection{SLF}

The tendency to describe SLF as a whole entity is generally proper to more technical studies, which address the different properties of dMRI methodologies. Within such studies, the detailed description of the WM bundles' anatomical characteristics is generally out of scope, and a broad characterization is usually sufficient (e.g., De Santis et al., 2014; Garyfallidis et al., 2018; Toga et al., 2006). The need to segment the SLF into sub-components arises when confronting the extent of its wiring.

\subsubsection{SLF I-III (Figure 7)}

The most commonly used nomenclature of SLF sub-components upholds three tracts running between the frontal and parietal lobes. They have classically been denoted with roman numerals from I to III, standing for their location on the sagittal plane following a dorsal to ventral distribution (Figure 7). This nomenclature was first introduced in monkeys by Petrides and Pandya (1984) via the autoradiographic technique. They described the presence of three tracts projecting from various areas of the posterior parietal region to the frontal cortex following an organization into distinct longitudinally running bundles and labeled as SLF I-III in an overall figure. This exact definition was therefore endorsed in non-human primate studies (Schmahmann et al., 2007). Makris et al. (2005) were probably the firsts to propose SLF's subdivision into three components in humans. It was then commonly accepted and corroborated based on ex-vivo (Monroy-Sosa et al., 2019; Yagmurlu et al., 2016) and neuroimaging (Kamali 
et al., 2014; Rojkova et al., 2016) evidence, and remained quite unvaried. Schurr et al. (2020) stress that this SLF segmentation is achievable not only through the study of the tract's macroanatomical properties, such as its course or sites of termination, but also via specific analysis of MRI-derived parameters. They could isolate SLF III from the rest of the SLF by comparing its fractional anisotropy (FA) values and relaxation times with those of SLF II. Thus, the microstructural properties of the sub-tracts support the SLF subdivision.

Considering evidences coming from DES studies (see also Duffau (2015)), the SLF would subserve two main functional domains: a dorsal network, broadly represented by SLF II, allows the integration of sensory visual and motor inputs (Sarubbo et al., 2020a; Thiebaut de Schotten, 2005), while a more ventral pathway corresponding to SLF III supports speech articulatory network (Duffau et al., 2003; Maldonado et al., 2011; Sarubbo et al., 2020a, 2016, 2015, 2013a; Zacà et al., 2018). More in detail, SLF II in the right hemisphere has been related to spatial awareness (Sarubbo et al., 2020a) and to the symmetrical processing of the visual scene (Thiebaut de Schotten, 2005), while SLF III in the left hemisphere has been implicated in verbal short term memory, with the role of conveying information about items' order (Papagno et al., 2017). While classical studies defined the existence of a visuo-spatial rightlateralized attentional network, Thiebaut de Schotten et al. (2011a) suggest that fronto-parietal connections in both hemispheres subserve visuo-spatial attentional skills, with a right hemisphere dominance in only part of the population. Further studies proposed that other cognitive skills might be mediated by the SLF. For instance, Herbet et al. (2014)'s lesion mapping study reports an implication of fronto-parietal connections in social cognition, while Spena et al. (2006) relate the SLF with the coordination of body posture and spatially-oriented actions via intra-operative DES mapping.

The putative description of SLF proposed by Makris et al. (2005)'s DTI study asserts that the most dorsal component of this bundle, SLF I, would connect the superior parietal lobule (SPG) and the precuneus ( $\mathrm{PrCu})$ to the dorsal part of the posterior superior frontal gyrus (SFG) including the supplementary motor area (SMA). This characterization was later reproduced with the use of the same technique: Jang and Hong (2012) stressed the involvement of the superior and medial parietal cortices ( $\mathrm{SPG}, \mathrm{PrCu})$ on one side and of the SMA and the dorsal premotor area (equivalent function-based nomenclature to the dorsal part of the posterior SFG as proposed by (Makris et al., 2005)) on the other side; Kamali et al. (2014) proposed this same definition again, although condensing the description of the posterior sites of termination of the tract in the SPG definition. The tensor model's desertion in favor of probabilistic tractography 
strengthened the finding of SLF I originating in PrCu and SPG. At the same time, it established, with the adhesion to a gyral nomenclature, that this subtracts' rostral terminal area should be represented by SFG (Rojkova et al., 2016). Within this methodological context, Thiebaut de Schotten et al. (2011), which sided the tractography reconstruction of the SLF with data from ex-vivo dissection, and Thiebaut de Schotten et al. (2012) complemented the definition of a tract connecting SPG and PrCu to the SFG by advancing the hypothesis of a possible extension to the anterior part of the cingulate gyrus. Two ensuing studies comparing DTI data with anatomical dissections replicated the presence of SLF I anterior terminations in the anterior cingulate gyrus and limited the implication of SFG only to its medial part (Baydin et al., 2017; Yagmurlu et al., 2016). One further study using this comparative approach (Güngör et al., 2017) specifies that such SLF I component would connect the superior part of the $\mathrm{PrCu}$ to anterior SFG.

Makris et al. (2005) defined SLF II as a connection linking AG to prefrontal regions (BAs 46, 6, 8) that we consider here and later corresponding to MFG. This finding was partially replicated by another more recent DTI study (Kamali et al., 2014). Thiebaut de Schotten et al. (2012, 2011) modified this depiction of the bundle, asserting that it extends slightly more dorsally both in the frontal and in the parietal lobes : caudal endpoints of this sub-tract could be found not only in the AG but also within the anterior intermediate parietal sulcus (Thiebaut de Schotten et al., 2011a) or along the intraparietal sulcus (Thiebaut de Schotten et al., 2012), while anteriorly it would connect to posterior MFG and SFG. A subsequent study using the same technique for tractographic reconstruction (Rojkova et al., 2016) confirmed the involvement of posterior MFG and attributed the caudal endings of SLF II to the AG. A series of studies coupling fiber dissection and dMRI-based tractography introduced further variability in the tract description. Wang et al. (2016) supported the extension of caudal ending sites to more dorsal areas in the parietal lobe, arguing that occasionally connections to the SPG could be found. Still, while supporting the specification of the posterior part of MFG as a rostral terminal area for the tract, they advanced a new proposal stressing the contribution of dorsal PrCG. Yagmurlu et al. (2016) replicated the description of connections between AG and MFG. First, they described SLF II anterior extensions to the lateral frontopolar cortex and posterior extensions to the anterior part of the middle occipital gyrus (MOG). Conversely to the previous studies stressing the involvement of MFG only in its more posterior part, Güngör et al. (2017) and Monroy-Sosa et al. (2019) proposed the tract to connect AG to the anterior and middle MFG. Additionally, Güngör et al. (2017) suggested that SLF II fibers would also reach the IFG, ending in IFGop and IFGtri. 
SLF III is described as the most ventral and lateral component of the SLF. According to its classical description, it connects SMG with IFGop, but it also extends to the ventral part of BA 6 (PrCG) and the middle third of BA 46 (MFG) (Makris et al., 2005). While the delineation of connections reaching MFG was never corroborated, Kamali et al. (2014) via DTI and Wang et al. (2016) by comparing DSI with fiber microdissection tracked SLF III from SMG to the ventral PrCG and IFGop (in Kamali et al. (2014) BAs 6 and 44 respectively). Nonetheless, other studies did not provide a so-detailed description of the anterior sites of termination of the sub-tract: Thiebaut de Schotten et al. (2011) showed SLF III to connect the temporo-parietal junction (BA 40) to the IFG; Thiebaut de Schotten et al. (2012) defined it as a bundle running from the IPL (AG, SMG) to BAs 44, 45, 47 (IFG); Rojkova et al. (2016) assert that it links SMG to IFG. Using DTI and fiber dissection, Yagmurlu et al. (2016) and Monroy-Sosa et al. (2019) partially supported SLF III's original description, attributing its cortical sites of termination to SMG and IFGop.

\subsubsection{SLF additional subdivision (Figure 8)}

The first study investigating the perisylvian WM by coupling tractography and fiber dissection technique proposed that the SLF could be segmented into a horizontal segment and a vertical segment (Fernández-Miranda et al., 2008) (Figure 8). In addition to a direct frontotemporal pathway, namely the arcuate segment of the superior longitudinal fasciculus, the authors identified a frontoparietal tract, running between prefrontal areas corresponding nearly to MFG and the inferior parietal lobe, mainly represented by AG and SMG, and a parietotemporal connection, arising in this parietal area and proceeding downward towards posterior MTG and STG. The use of a more advanced dissection technique such as the cortex sparing approach developed by Martino et al. (2011) has provided a better delineation of the SLF cortical sites of termination within this theoretical framework. The SLF's horizontal segment was defined as connecting the frontal operculum (vPrCG, IFGop, IFGtri) to SMG. In contrast, the vertical segment would arise more posteriorly in the inferior parietal lobe, running between AG and the posterior part of MTG. Sarubbo et al. (2016)'s dissection study tracked the anterior $S L F$ from the ventral premotor cortex (vPrCG) and IFGop to both SMG and AG, while they found the posterior component of the SLF running from AG and SMG to the middle thirds of MTG and STG and the anterior segment of MOG. Vassal et al. (2016) combined DTI tractography and the study of cortical activation patterns via functional magnetic resonance imaging (fMRI) to map language-related WM fascicles. Their analysis supported the characterization of a frontoparietal component of the SLF connecting vPrCG and, in some 
cases, IFGop to SMG; the parieto-temporal segment was defined as a connection between AG (in some cases also SMG) and MTG (with a certain degree of extension to STG).

Further works provided an accurate description of the SLF's temporo-parietal connection and proposed an additional segmentation of this tract into subcomponents. Following the previous reports, Kamali et al. (2014)'s DTI study isolated a bundle of fibers arising in the posterior segment of STG and connecting to AG. Nonetheless, the authors assert that most of the fibers representing the temporoparietal $S L F$ proceed even more dorsally after reaching the level of IPL, heading towards the posterior portion of SPG. Therefore, they proposed distinguishing between the SLF TP-IPL, which represents the classical description of the tract, and the SLF TP-SPL, an additional component ascribable to the SLF system. Wu et al. (2016b) conducted both DSI and fiber dissection in the temporo-parieto-occipital region and supported the dissociation between these two temporo-parietal connections. In contrast with Kamali et al. (2014)'s proposal, they defined a fifth SLF component, or SLF V, encompassing only those fibers arising in the posterior portions of MTG and ITG and connecting to the SMG and AG. They even further subdivided SLF V into angular- or supramarginal-SLF-V depending on the fibers' sites of parietal terminations. Given that DES of this WM pathway produces anomia, the posterior portion of the SLF has been found to subserve visuo-semantic integration. These short fibers are therefore thought to be involved in access and retrieval of semantic information for language processing (Sarubbo et al., 2020b, 2015) in essential cortical territories (i.e. temporo-parietal junction) for the integration of sensory inputs in different modalities of language elaboration (Maffei et al., 2019, 2018; Sarubbo et al., 2015).

\section{General discussion}

With the historical perspective and a review of recent dissection and dMRI-based tractography studies, we have attempted to synthesize the current knowledge about the long association fibers that connect the frontal lobe to the parietal, occipital, and temporal lobes through the upper pathway. Such fibers currently belong to the SLF and/or AF and their different sub-components and constitute the SLS proposed by Mandonnet et al. (2018).

One may first point out that, although dMRI-based tractography and Klingler dissection are the current suitable alternatives for the study of the structural connectivity of the human brain at the macroscopic level, they are not perfect either. The earliest tractography algorithms based on the diffusion tensor model were only capable of distinguishing a single fiber 
population per voxel, providing a poor representation of the underlying fiber orientations in which tracking can terminate prematurely (Behrens et al., 2007). Representing the fiber orientations as a continuous function of the sphere, i.e., the fiber orientation distribution function (fODF) (Descoteaux et al., 2009; Tournier et al., 2007), led to better tractography algorithms able to track in WM regions with complex fiber architecture. Nonetheless, the neuroscience community has also explicitly recognized the pros and cons of these current tractography algorithms (Maier-Hein et al., 2017; Rheault et al., 2020b). Most of the algorithms produce tractograms containing $90 \%$ of a given set of ground truth bundles, but the same tractograms include at least four times more invalid than valid bundles. It involves applying some post-tractographic bundle segmentation relying on the bundle's original definition, emphasizing its considered anatomical definition. As we observed in the current review, the authors thus essentially used a manual bundle segmentation. Since the pioneering studies of Catani et al. (2002) and Wakana et al. (2007), this approach corresponds to carefully delineating ROIs or using atlas-based ROIs on which applying sets of rules to isolate a bundle of interest (e.g., Rojkova et al., 2016; Thiebaut de Schotten et al., 2011b; Zhang et al., 2010). The primary bias of such an approach relies on the definition, size, and location of the ROIs used to extract a specific bundle. It has also recently been shown that the same anatomical definition, on the same tractogram, from different rater produced slightly different results (Rheault et al., 2020a). The classic dissection approach described by Klingler (1935)involves freezing/defrosting formalin-fixed brains to isolate a fiber tract by removing the cortex and overlying WM. Nevertheless, an extensive removal of brain tissue prevents the description of tracts in terms of cortical landmarks, which complicates the analysis of cortical terminations. The cortex-sparing Klingler dissection approach proposed by Martino et al. (2011) in which removal of brain tissue is kept to a minimum to preserve the cortex and relationships within the brain until the end of the dissection, allows following the fibers until their cortical termination to better analyze the cortical anatomical connectivity. Moreover, Klingler dissection requires postmortem specimens, on which inadequate tissue fixation and preservation can hamper the dissection's accuracy. Another limitation is that a dissectionist will inevitably make a subjective observation and strictly follow a reproducible protocol. Interestingly, electron microscopy has confirmed that the myelin sheaths are preserved despite the freezing/defrosting process; therefore, the axonal integrity is conserved during the dissection, which supports the reliability of Klingler's approach to explore the brain WM (Zemmoura et al., 2016). Applying the photogrammetry technique, which provides an accurate reconstruction of a 3D model from multiple stereoscopic images, to the macrostructural analysis of the human brain connectivity is also a promising 
application of the cortex-sparing Klingler dissection approach (De Benedictis et al., 2018). It permits the reproduction of a layer-by-layer dissection of the WM tracts and visualizes the anatomic results from a real brain specimen into an interactive explorable 3D model. Although unsatisfactory taken on their own, these different approaches are very complementary. They must be combined in the process of validation of the anatomy of WM fibers on a macroscopic scale, as demonstrated in recent years (e.g., De Benedictis et al., 2016; Hau et al., 2017; Maffei et al., 2018; Panesar et al., 2018; Sarubbo et al., 2019; Wang et al., 2016).

Since their first descriptions in the 19th century, the characterization of AF and SLF as a single entity or not has evolved together with the discovery and the improvement of in-vivo segmentation and ex-vivo dissection techniques. Still, this advancement did not strictly coincide with the refinement of the related information, mainly due to a lack of systematicity in how knowledge has been categorized. As a matter of fact, there is no agreement upon the definition of these WM pathways, and the variability across the different descriptions fundamentally relies on the lack of ground truth for the conceptualization of knowledge. For instance, some of the studies representing the backbone of the SLS literature labeled their findings differently while still pointing at the same concept. The tripartite subdivision of the AF (Catani et al., 2005) can be re-examined in light of the segmentation of perisylvian WM tracts advanced by FernándezMiranda et al. (2008). Both standpoints stress the existence of three main pathways connecting frontal, parietal, and temporal lobes, but while Catani et al. (2005) applied their segmentation to the AF, Fernández-Miranda et al. (2008) implemented this specification to a broader system. Indeed, they assert that the horizontal and vertical segments (respectively anterior and posterior segments as proposed by Catani et al. (2005)) can instead be ascribed to the SLF. It is reasonable to assume that both studies described the same structures, but the information from the two standpoints was hardly comparable due to different terminologies. In light of more recent characterizations of the SLS, which support its segmentation into SLF I-III and AF, it could be asserted that the horizontal or anterior segment is nothing but a ventral component of the SLF, probably part of the SLF III (Mandonnet et al., 2018; Yagmurlu et al., 2016; Zemmoura et al., 2014). Only one tractography study reported the coexistence of SLF I-III and the anterior segment of the AF on top of the description of the direct connection between frontal and temporal lobes, namely the long segment of the AF (Rojkova et al., 2016); nonetheless, this finding remains isolated. 
The matter becomes even more complicated when addressing the status of the posterior or vertical segment of AF. The multiple nomenclatures attributed to the long-range association pathways between parietal and temporal lobes closely mirror how the debate concerning the SLS's hierarchical organization has evolved. First defined as the posterior segment of the AF (Catani et al. (2005), later supported by Barrick et al. (2006), Forkel et al. (2020) and Lawes et al. (2008)), its affiliation to a more extensive system was introduced that same year with the proposal of the SLF I-III segmentation (Makris et al., 2005). Indeed, within the theoretical framework stressing the exact overlap of the concepts related to arcuate fasciculus and superior longitudinal fasciculus, Makris et al. (2005) name this bundle the vertical portion of the AF, which is in turn defined as the fourth component of the SLF. As mentioned above, FernándezMiranda et al. (2008) ascribe this connection to the SLF; this standpoint was later extensively supported, leading to the employment of the labels temporoparietal segment of the SLF (Kamali et al., 2014; Vassal et al., 2016), SLF V (Wu et al., 2016b) and indirect posterior SLF (Sarubbo et al., 2016). Further studies applying the tripartite subdivision to a broader ensemble of WM fibers delineated the posterior segment within the entirety of perisylvian connections, without addressing its relationship with the rest of the system (Gharabaghi et al., 2009; Martino et al., 2013; Zemmoura et al., 2014). It is also noteworthy that a relevant number of studies addressing perisylvian WM tracts neglected this parieto-temporal connection in their description (Güngör et al., 2017; Rojkova et al., 2016; Thiebaut de Schotten et al., 2012; Wang et al., 2016; Yagmurlu et al., 2016). Thus, the issues related to adopting different categorizations for the definition of the same anatomical structures also open a more general controversy, questioning the fibers' affiliation to which system. Frey et al. (2008) described connections running from the posterior temporal lobe to ventral parietal regions as part of the middle longitudinal fasciculus (MdLF). Based on different hemispheric lateralization patterns compared to both SLF and AF, Panesar et al. (2019) stress the complete independence of this component from the MdLF, proposing to name it the temporo-parietal aslant tract.

At this level of discussion, another matter of debate concerns the nature of SLF I. Even though most of the literature agrees on describing fibers connecting dorsomedial parietal and frontal cortices as the most dorsal component of the SLF (e.g., Baydin et al., 2017; Jang and Hong, 2012; Kamali et al., 2014), Wang et al. (2016) propose that this connection should be traced back to the cingulum bundle. Indeed, while their DSI data showed a group of fibers laying just dorsal to the cingulum fibers and supposedly representing the SLF I, this sub-tract could not be isolated via dissection in any of their specimens. To account for the inability to 
extract this component ex-vivo, Wang et al. (2016) stress that the spatial relationship of SLF I with the nearby WM tracts justifies its attribution to the cingulum bundle itself or at least to the cingulum system rather than to the SLF ensemble. This view was further supported by Wu et al. (2016a), which segmented the cingulum bundle in its subcomponents through the use of the same DSI-fiber dissection comparative approach. They assert that previous studies erroneously attributed some fibers of the cingulum to the SLF I and that these same fibers represent the fourth component of the cingulum $(C B-I V)$.

On the other hand, a recent dissection study by Komaitis et al. (2019) defines the guidelines for separating SLF I fibers from those of the cingulum. It proposes a transition point between the two bundles at the anterior paracentral lobule level, emphasizing their discreteness. Nonetheless, the authors state the inability to identify a direct anatomical relationship of the SLF I with the other SLF components. A dissection study by Maldonado et al. (2012) proposed a more extreme viewpoint on the matter, advancing that previous tractographic reconstructions of this fiber bundle are instead artifacts. According to this standpoint, SLF I would not exist, or at least it would not reflect the typical characterization attributed classically to it: at this dorsomedial level, no long-range horizontally oriented fronto-parietal connection could be found, and the connectivity between the two lobes would be provided by a series of short Ushaped intergyral tracts. Indeed, according to the authors, the presence of transversal sulci conflicts with the existence of a continuous horizontal bundle of fibers, at least until the level of the base of the gyri. These short-range connections themselves might represent a confusing factor for dMRI, accounting for a possible artefactual reconstruction of SLF I (Maldonado et al., 2012).

The AF and the SLF are known to be mainly involved in the dorsal phono-articulatory language loop and in sensory-motor integration processes (Maldonado et al., 2011; Sarubbo et al., 2020a, 2015; Thiebaut de Schotten, 2005). Still, as outlined in a recent review by Forkel et al. (2020a) and as briefly addressed by the present work, it is not possible to trace a precise oneto-one correlation between a WM structure and the derived cognitive function across studies, being function a property arising from the interplay of different cortices and mediated by WM tracts. Similarly to the controversies described for the structural definition of the SLS, the adoption of different nomenclatures and the application of different partitionings within the system does not encourage comparisons between functional studies. For instance, functions related to ventral fronto-parietal connections can be attributed to the anterior segment of the AF or to SLF III - or even anterior segment of the SLF - depending on whether the authors stick to 
the tripartite subdivision of the AF or to the SLF I-III nomenclature (Papagno et al., 2017; Sarubbo et al., 2020a, 2016, 2015). Moreover, the assumption of a certain partonomy of the system can lead to misunderstandings when describing the findings of a study. It is the case of Herbet et al. (2014), which stresses the involvement of the AF and the SLF in mentalizing: although they are described as two separate components, both the AF and the SLF are reported to be fronto-parietal connections.

Based on the currently available neuroimaging and ex-vivo dissection techniques, none of the previously advanced nomenclatures should be defined as improper. When analyzing the upper WM pathways of the perisylvian region, different descriptions were made by various reports, depending on the aim of the study itself. Thus, all the taxonomies proposed to define this system of WM fibers can be justified based on the model that drove the observation. An illustration of the legitimacy of all the proposed classification given a data-driven approach can be found in Goryaynov et al. (2017)'s study, which carried out a comparison between fiber dissection and DTI-based tractography. While reporting the results of their ex-vivo analysis of the SLF and the AF, the authors claim: "Ambiguous data were obtained regarding their segmentation: a two-segment structure (SLFh and SLFv) was found in most (10/12) specimens, while a three-segment structure was revealed in the other (2/12) specimens (identified SLF II and SLF III). In the arcuate fascicle, the ventral and dorsal segments were successfully identified in 2/12 cases (1 left and 1 right), whereas identification failed in the other cases." Even though the specimens were fixed and dissected according to the same protocol, different possible segmentations were attributed to the SLS, stressing that any type of categorization can potentially be attributed to this ensemble of fibers. And again: "There are three main approaches to [the SLF] dissection: 1) a two-segment approach, according to which, after the removal of the cortex and short fibers, first its vertical part (SLFv) is isolated from the thickness of the superior temporal gyrus and then the horizontal part (SLFh) is isolated deep in the middle frontal gyrus; 2) a three-segment one, described in the presented protocol of lateral dissection; 3 ) in the form of an indivisible complex of the superior longitudinal fascicle with the arcuate fascicle.". Thus, the approach that we assume towards the data may shape the characteristics we attribute to them. But this study by Goryaynov et al. (2017) also reveals a point hardly ever addressed in all the reports we have reviewed: the difficulty in extracting the different bundles or their various supposed components would be in part related to individual variability. Indeed, different factors, such as gender (Thiebaut de Schotten et al., 2011b), age (Rojkova et al., 2016) and manual preference (Howells et al., 2018), are closely related to WM architecture, and can 
possibly be linked to an enhancement or a reduction of certain structural features in one of the two hemispheres as well as in one individual compared to another. The issue of inter-subjects variability can more easily be addressed with the adoption of a more comprehensive approach for the characterization of the WM rather than by forcing a specific partitioning to extract defined components from a system.

Numerous studies recognize the confusion which characterizes the SLS literature (Dick and Tremblay, 2012; Mandonnet et al., 2018; Panesar and Fernandez-Miranda, 2019), and various attempts to systematize all the different findings have been made. In particular, we acknowledge a recent work by Nakajima et al. (2019), which proposes a new function-based nomenclature of the SLF (including the AF as its subcomponent). From our standpoint, the definition of the function mediated by a WM pathway is, no doubt, valuable complementary information to its structural description. Still, it cannot possibly represent the cornerstone for the study of brain connectivity. As mentioned-above, function is an attribute made possible by the existence of a structural link between multiple brain areas, and different functions can arise from their interplay. Therefore, the only systematical way to describe the human brain WM pathways consists of considering the constraints that the anatomy itself establishes. Moreover, the definition of a tract based on macroscopic landmarks represents the easiest and most accessible way for its characterization: the organization into sulci and gyri of a single brain can readily be inferred in a non-invasive way from a T1 image and ex-vivo with the cortex-sparing Klingler dissection approach (Martino et al., 2011)

\section{Proposal for a medio-lateral palisading arrangement of the SLS}

The SLS encompasses short- and long-range fibers that connect the frontal cortex with the parietal, temporal, and occipital lobes while passing above the superior limiting sulcus of the insula. The present literature review shows that the SLS covers the entire convexity of the hemisphere from the deepest and most medial part (near the cingulum) to the most lateral and superficial part. As observed with Klingler's dissections of the association pathways (e.g., Tabula 6 of Klingler in Figure 3; and see also Sarubbo et al., 2016; Wang et al., 2016)), the SLS presents a regular topography which was recently well-designated by Mortazavi et al. (2018) as hierarchic palisades of fiber structure. It does not appear from the multitude of studies already carried out that specific anatomical landmarks, similar to the stems of IFOF or UF (Hau et al., 2016; Martino et al., 2010; Sarubbo et al., 2013b), can be defined to distinguish the different SLFI-III or AF type components. They instead appear as a continuum of reciprocal 
connections between each gyrus, arranged accordingly to a medio-lateral homotopic topography without necessarily determining separated bundles (Figure 9). In other words, only the sulcal anatomy of the frontal, parietal, occipital, and temporal cortices would allow a specific separation within the SLS, which would overwhelmingly respect a palisade organization following a medio-lateral longitudinal homotopy around the circular sulcus of the insula. As schematically illustrated in Figure 9, the medial part of the superior frontal cortex would connect the medial superior parietal cortex, namely the deepest part of the SLS. In the same vein, the most lateral part of the inferior frontal cortex connects the most lateral part of the inferior parietal cortex (SMG, AG).

Interestingly, it has been also demonstrated that the fiber pathway system is well approximated by a lamellar vector field (Galinsky and Frank, 2016). Consistent with the laminar origin of cortico-cortical connections demonstrated in non-human primates at the mesoscopic level (Barbas, 1986), this tendency for lamellarity in human brain fiber pathways is currently what the dissectionists "à la Klingler" are making at the macroscopic level by progressing sequentially in a latero-medial direction along the hierarchic palisades of fiber structure (Martino et al., 2011; Sarubbo et al., 2016). Considering the SLS with such a topographical arrangement does not necessarily imply that the multiple previous SLF and AF descriptions are erroneous but somewhat incomplete. They all sought to extract distinct bundles between specific regions as if there were unconnected spaces between certain frontal regions and the brain's posterior regions.

Our proposal for a palisading arrangement of the SLS warrants a more in-depth investigation. One may consider the very numerous neural tracing studies obtained in nonhuman primates for the description of the SLF and AF (e.g. Schmahmann and Pandya, 2006). It may also include the most recent works dealing with dissection and tractography in the nonhuman primate (e.g. Decramer et al., 2018; Mars et al., 2019; Thiebaut de Schotten et al., 2012). Another systematic study, similar and complementary to the one we propose here for the human brain, should be devoted to all the data on non-human primates to be sufficiently complete.

\section{Conclusion}

The current state of the SLS description, which encompasses both SLF and AF, does not allow for describing its general hodology issued of all the related knowledge produced. It is clear that the inability to reach a consensus on its anatomical description (i.e., course and cortical sites of termination) is only the most evident issue in this respect and that the main 
hindrance to overcome is related to the lack of a univocal rule for the conceptualization of anatomical knowledge. No significant progress in the field can be envisaged until a shared paradigm is established by mutual consent. The step forward that we aim to promote does not require the falsification of any of the previously advanced nomenclatures. We then propose considering the association pathways' organization similar to the topographical arrangement of commissural and projection pathways, namely less as individualized fascicles than respecting an orderly and continuous wiring diagram with a layer-based topography. A better and complete understanding of the precise organization of the connectivity of WM association pathways is fundamental to a better understanding of brain function and their clinical and neurosurgical applications. 


\section{References}

Agrawal, A., Kapfhammer, J.P., Kress, A., Wichers, H., Deep, A., Feindel, W., Sonntag, V.K.H., Spetzler, R.F., Preul, M.C., 2011. Josef Klingler's Models of White Matter Tracts: Influences on Neuroanatomy, Neurosurgery, and Neuroimaging: Neurosurgery 69, 238-254. https://doi.org/10.1227/NEU.0b013e318214ab79

Barbas, H., 1986. Pattern in the laminar origin of corticocortical connections. J. Comp. Neurol. 252, 415-422.

Barrick, T.R., Lawes, I.N., Mackay, C.E., Clark, C.A., 2006. White Matter Pathway Asymmetry Underlies Functional Lateralization. Cereb. Cortex 17, 591-598. https://doi.org/10.1093/cercor/bhk004

Baydin, S., Gungor, A., Tanriover, N., Baran, O., Middlebrooks, E.H., Rhoton, A.L., 2017. Fiber Tracts of the Medial and Inferior Surfaces of the Cerebrum. World Neurosurg. 98, 34-49. https://doi.org/10.1016/j.wneu.2016.05.016

Behrens, T.E.J., Berg, H.J., Jbabdi, S., Rushworth, M.F.S., Woolrich, M.W., 2007. Probabilistic diffusion tractography with multiple fibre orientations: What can we gain? NeuroImage 34, 144-155. https://doi.org/10.1016/j.neuroimage.2006.09.018

Bernal, B., Altman, N., 2010. The connectivity of the superior longitudinal fasciculus: A tractography DTI study. Magn. Reson. Imaging 28, 217-225. https://doi.org/10.1016/j.mri.2009.07.008

Burdach, K.F., 1822. Vom Baue und Leben des Gehirns. Leipzig: Dyk.

Catani, M., Howard, R.J., Pajevic, S., Jones, D.K., 2002. Virtual in Vivo interactive dissection of white matter fasciculi in the human brain. NeuroImage 17, 77-94. https://doi.org/10.1006/nimg.2002.1136

Catani, M., Jones, D.K., Ffytche, D.H., 2005. Perisylvian language networks of the human brain. Ann. Neurol. 57, 8-16. https://doi.org/10.1002/ana.20319

Conner, A.K., Briggs, R.G., Rahimi, M., Baker, C.M., Burks, J.D., Glenn, C.A., Battiste, J.D., 2018. A CONNECTOMIC ATLAS OF THE HUMAN CEREBRUM SUPPLEMENT A Connectomic Atlas of the Human Cerebrum - Chapter 10 : Tractographic Description of the Superior Longitudinal Fasciculus. Connect. Atlas Hum. Cerebrum Suppl. 15, 407-422. https://doi.org/10.1093/ons/opy264

De Benedictis, A., Duffau, H., Paradiso, B., Grandi, E., Balbi, S., Granieri, E., Colarusso, E., Chioffi, F., Marras, C.E., Sarubbo, S., 2014. Anatomo-functional study of the temporo-parieto-occipital region: Dissection, tractographic and brain mapping evidence from a neurosurgical perspective. J. Anat. 225, 132-151. https://doi.org/10.1111/joa.12204

De Benedictis, A., Nocerino, E., Menna, F., Remondino, F., Barbareschi, M., Rozzanigo, U., Corsini, F., Olivetti, E., Marras, C.E., Chioffi, F., Avesani, P., Sarubbo, S., 2018. Photogrammetry of the Human Brain: A Novel Method for Three-Dimensional Quantitative Exploration of the Structural Connectivity in Neurosurgery and Neurosciences. World Neurosurg. 115, e279-e291. https://doi.org/10.1016/j.wneu.2018.04.036

De Benedictis, A., Petit, L., Descoteaux, M., Marras, C.E., Barbareschi, M., Corsini, F., Dallabona, M., Chioffi, F., Sarubbo, S., 2016. New insights in the homotopic and heterotopic connectivity of the frontal portion of the human corpus callosum revealed by microdissection and diffusion tractography: Homo- and Hetero-Topic FrontoCallosal Connectivity. Hum. Brain Mapp. 37, 4718-4735. https://doi.org/10.1002/hbm.23339

Decramer, T., Swinnen, S., van Loon, J., Janssen, P., Theys, T., 2018. White matter tract anatomy in the rhesus monkey: a fiber dissection study. Brain Struct. Funct. 223, 3681-3688. https://doi.org/10.1007/s00429-018-1718-x 
Dejerine, J., Dejerine-Klumpke, A., 1901. Anatomie des centres nerveux. Tome 2. Paris: Rueff et Cie.

Dejerine, J., Dejerine-Klumpke, A., 1895. Anatomie des centres nerveux. Tome 1. Paris: Rueff et Cie.

Descoteaux, M., Deriche, R., Knosche, T.R., Anwander, A., 2009. Deterministic and Probabilistic Tractography Based on Complex Fibre Orientation Distributions. IEEE Trans. Med. Imaging 28, 269-286. https://doi.org/10.1109/TMI.2008.2004424

Destrieux, C., Terrier, L.M., Andersson, F., Love, S.A., Cottier, J.-P., Duvernoy, H., Velut, S., Janot, K., Zemmoura, I., 2017. A practical guide for the identification of major sulcogyral structures of the human cortex. Brain Struct. Funct. 222, 2001-2015. https://doi.org/10.1007/s00429-016-1320-z

Dick, A.S., Tremblay, P., 2012. Beyond the arcuate fasciculus: consensus and controversy in the connectional anatomy of language. Brain 135, 3529-3550. https://doi.org/10.1093/brain/aws222

Duffau, H., 2015. Stimulation mapping of white matter tracts to study brain functional connectivity. Nat. Rev. Neurol. 11, 255-265. https://doi.org/10.1038/nrneurol.2015.51

Duffau, H., Gatignol, P., Denvil, D., Lopes, M., Capelle, L., 2003. The articulatory loop: study of the subcortical connectivity by electrostimulation: NeuroReport 14, 20052008. https://doi.org/10.1097/00001756-200310270-00026

Fernández-Miranda, J.C., Rhoton, A.L., Álvarez-Linera, J., Kakizawa, Y., Choi, C., De Oliveira, E.P., 2008. Three-dimensional microsurgical and tractographic anatomy of the white matter of the human brain. Neurosurgery 62, 989-1028. https://doi.org/10.1227/01.NEU.0000297076.98175.67

Fernández-Miranda, J.C., Wang, Y., Pathak, S., Stefaneau, L., Verstynen, T., Yeh, F.-C., 2015. Asymmetry, connectivity, and segmentation of the arcuate fascicle in the human brain. Brain Struct. Funct. 220, 1665-1680. https://doi.org/10.1007/s00429-014-07517

Forkel, S.J., Friedrich, P., de Schotten, M.T., Howells, H., 2020a. White matter variability, cognition, and disorders: a systematic review. https://doi.org/10.1101/2020.04.22.20075127

Forkel, S.J., Rogalski, E., Drossinos Sancho, N., D’Anna, L., Luque Laguna, P., Sridhar, J., Dell'Acqua, F., Weintraub, S., Thompson, C., Mesulam, M.-M., Catani, M., 2020b. Anatomical evidence of an indirect pathway for word repetition. Neurology 94, e594e606. https://doi.org/10.1212/WNL.0000000000008746

Frey, S., Campbell, J.S.W., Pike, G.B., Petrides, M., 2008. Dissociating the Human Language Pathways with High Angular Resolution Diffusion Fiber Tractography. J. Neurosci. 28, 11435-11444. https://doi.org/10.1523/JNEUROSCI.2388-08.2008

Galinsky, V.L., Frank, L.R., 2016. The Lamellar Structure of the Brain Fiber Pathways. Neural Comput. 28, 2533-2556. https://doi.org/10.1162/NECO_a_00896

Geschwind, N., 1967. Wernicke's Contribution to the Study of Aphasia. Cortex 3, 449-463. https://doi.org/10.1016/S0010-9452(67)80030-3

Gharabaghi, A., Kunath, F., Erb, M., Saur, R., Heckl, S., Tatagiba, M., Grodd, W., Karnath, H.-O., 2009. Perisylvian white matter connectivity in the human right hemisphere. BMC Neurosci. 10, 15. https://doi.org/10.1186/1471-2202-10-15

Glasser, M.F., Rilling, J.K., 2008. DTI Tractography of the Human Brain's Language Pathways. Cereb. Cortex 18, 2471-2482. https://doi.org/10.1093/cercor/bhn011

Goryaynov, S.A., Kondrashov, A.V., Gol'dberg, M.F., Batalov, A.I., Sufianov, R.A., Zakharova, N.E., Pronin, I.N., Gol'bin, D.A., Zhukov, V.Yu., Dobrovol'skiy, G.F., Shelyakin, S.Yu., Vorob'ev, V.N., Dadykin, S.S., Potapov, A.A., 2017. Long association tracts of the human white matter: an analysis of 18 hemisphere dissections 
and in vivo HARDI-CSD tractography. Vopr. Neirokhirurgii Im. NN Burdenko 81, 13. https://doi.org/10.17116/neiro201780713-25

Güngör, A., Baydin, S., Middlebrooks, E.H., Tanriover, N., Isler, C., Rhoton, A.L., 2017. The white matter tracts of the cerebrum in ventricular surgery and hydrocephalus. J. Neurosurg. 126, 945-971. https://doi.org/10.3171/2016.1.JNS152082

Hau, J., Sarubbo, S., Houde, J.C., Corsini, F., Girard, G., Deledalle, C., Crivello, F., Zago, L., Mellet, E., Jobard, G., Joliot, M., Mazoyer, B., Tzourio-Mazoyer, N., Descoteaux, M., Petit, L., 2017. Revisiting the human uncinate fasciculus, its subcomponents and asymmetries with stem-based tractography and microdissection validation. Brain Struct. Funct. 222, 1645-1662. https://doi.org/10.1007/s00429-016-1298-6

Hau, J., Sarubbo, S., Perchey, G., Crivello, F., Zago, L., Mellet, E., Jobard, G., Joliot, M., Mazoyer, B.M., Tzourio-Mazoyer, N., Petit, L., 2016. Cortical Terminations of the Inferior Fronto-Occipital and Uncinate Fasciculi: Anatomical Stem-Based Virtual Dissection. Front. Neuroanat. 10. https://doi.org/10.3389/fnana.2016.00058

Herbet, G., Lafargue, G., Bonnetblanc, F., Moritz-Gasser, S., de Champfleur, N.M., Duffau, H., 2014. Inferring a dual-stream model of mentalizing from associative white matter fibres disconnection. Brain 16.

Howells, H., Thiebaut de Schotten, M., Dell'Acqua, F., Beyh, A., Zappalà, G., Leslie, A., Simmons, A., Murphy, D.G., Catani, M., 2018. Frontoparietal Tracts Linked to Lateralized Hand Preference and Manual Specialization. Cereb. Cortex 28, 24822494. https://doi.org/10.1093/cercor/bhy040

Jang, S.H., Hong, J.H., 2012. The anatomical characteristics of superior longitudinal fasciculus I in human brain: Diffusion tensor tractography study. Neurosci. Lett. 506, 146-148. https://doi.org/10.1016/j.neulet.2011.10.069

Jeurissen, B., Descoteaux, M., Mori, S., Leemans, A., 2019. Diffusion MRI fiber tractography of the brain. NMR Biomed. 32, e3785. https://doi.org/10.1002/nbm.3785

Kamali, A., Flanders, A.E., Brody, J., Hunter, J.V., Hasan, K.M., 2014. Tracing superior longitudinal fasciculus connectivity in the human brain using high resolution diffusion tensor tractography. Brain Struct. Funct. 219, 269-281. https://doi.org/10.1007/s00429-012-0498-y

Klingler, J., 1935. Erleichterung des makroskopischen Praeparation des Gehirns durch den Gefrierprozess. Schweiz. Arch. Für Neurol. Psychiatr. 36, 247-256.

Komaitis, S., Skandalakis, G.P., Kalyvas, A.V., Drosos, E., Lani, E., Emelifeonwu, J., Liakos, F., Piagkou, M., Kalamatianos, T., Stranjalis, G., Koutsarnakis, C., 2019. Dorsal component of the superior longitudinal fasciculus revisited: novel insights from a focused fiber dissection study. J. Neurosurg. 1-14. https://doi.org/10.3171/2018.11.JNS182908

Kuhn, T.S., 1996. The structure of scientific revolutions, 3rd ed. ed. University of Chicago Press, Chicago, IL.

Lawes, I.N.C., Barrick, T.R., Murugam, V., Spierings, N., Evans, D.R., Song, M., Clark, C.A., 2008. Atlas-based segmentation of white matter tracts of the human brain using diffusion tensor tractography and comparison with classical dissection. NeuroImage 39, 62-79. https://doi.org/10.1016/j.neuroimage.2007.06.041

Leemans, A., 2019. Diffusion MRI of the brain: The naked truth. NMR Biomed. 32, e4084. https://doi.org/10.1002/nbm.4084

Ludwig, E., Klingler, J., 1956. Atlas cerebri humani: Der innere Bau des Gehirns dargestellt auf Grund makroskopischer Präparate. The inner structure of the brain demonstrated on the basis of macroscopical preparations. Little, Brown.

Maffei, C., Jovicich, J., De Benedictis, A., Corsini, F., Barbareschi, M., Chioffi, F., Sarubbo, S., 2018. Topography of the human acoustic radiation as revealed by ex vivo fibers 
micro-dissection and in vivo diffusion-based tractography. Brain Struct. Funct. 223, 449-459. https://doi.org/10.1007/s00429-017-1471-6

Maffei, C., Sarubbo, S., Jovicich, J., 2019. A Missing Connection: A Review of the Macrostructural Anatomy and Tractography of the Acoustic Radiation. Front. Neuroanat. 13, 27. https://doi.org/10.3389/fnana.2019.00027

Maier-Hein, K.H., Neher, P.F., Houde, J.-C., Côté, M.-A., Garyfallidis, E., Zhong, J., Chamberland, M., Yeh, F.-C., Lin, Y.-C., Ji, Q., Reddick, W.E., Glass, J.O., Chen, D.Q., Feng, Y., Gao, C., Wu, Y., Ma, J., He, R., Li, Q., Westin, C.-F., DeslauriersGauthier, S., González, J.O.O., Paquette, M., St-Jean, S., Girard, G., Rheault, F., Sidhu, J., Tax, C.M.W., Guo, F., Mesri, H.Y., Dávid, S., Froeling, M., Heemskerk, A.M., Leemans, A., Boré, A., Pinsard, B., Bedetti, C., Desrosiers, M., Brambati, S., Doyon, J., Sarica, A., Vasta, R., Cerasa, A., Quattrone, A., Yeatman, J., Khan, A.R., Hodges, W., Alexander, S., Romascano, D., Barakovic, M., Auría, A., Esteban, O., Lemkaddem, A., Thiran, J.-P., Cetingul, H.E., Odry, B.L., Mailhe, B., Nadar, M.S., Pizzagalli, F., Prasad, G., Villalon-Reina, J.E., Galvis, J., Thompson, P.M., Requejo, F.D.S., Laguna, P.L., Lacerda, L.M., Barrett, R., Dell'Acqua, F., Catani, M., Petit, L., Caruyer, E., Daducci, A., Dyrby, T.B., Holland-Letz, T., Hilgetag, C.C., Stieltjes, B., Descoteaux, M., 2017. The challenge of mapping the human connectome based on diffusion tractography. Nat. Commun. 8, 1349. https://doi.org/10.1038/s41467-01701285-x

Makris, N., Kennedy, D.N., McInerney, S., Sorensen, A.G., Wang, R., Caviness, V.S., Pandya, D.N., 2005. Segmentation of Subcomponents within the Superior Longitudinal Fascicle in Humans: A Quantitative, In Vivo, DT-MRI Study. Cereb. Cortex 15, 854-869. https://doi.org/10.1093/cercor/bhh186

Maldonado, I.L., Mandonnet, E., Duffau, H., 2012. Dorsal Fronto-Parietal Connections of the Human Brain: A Fiber Dissection Study of Their Composition and Anatomical Relationships. Anat. Rec. Adv. Integr. Anat. Evol. Biol. 295, 187-195. https://doi.org/10.1002/ar.21533

Maldonado, I.L., Moritz-Gasser, S., Duffau, H., 2011. Does the left superior longitudinal fascicle subserve language semantics? A brain electrostimulation study. Brain Struct. Funct. 216, 263-274. https://doi.org/10.1007/s00429-011-0309-X

Mandonnet, E., Sarubbo, S., Petit, L., 2018. The nomenclature of human white matter association pathways: Proposal for a systematic taxonomic anatomical classification. Front. Neuroanat. 12, 1-14. https://doi.org/10.3389/fnana.2018.00094

Mars, R.B., O’Muircheartaigh, J., Folloni, D., Li, L., Glasser, M.F., Jbabdi, S., Bryant, K.L., 2019. Concurrent analysis of white matter bundles and grey matter networks in the chimpanzee. Brain Struct. Funct. 224, 1021-1033. https://doi.org/10.1007/s00429018-1817-8

Martino, J., De Witt Hamer, P.C., Berger, M.S., Lawton, M.T., Arnold, C.M., De Lucas, E.M., Duffau, H., 2013. Analysis of the subcomponents and cortical terminations of the perisylvian superior longitudinal fasciculus: A fiber dissection and DTI tractography study. Brain Struct. Funct. 218, 105-121. https://doi.org/10.1007/s00429-012-0386-5

Martino, J., de Witt Hamer, P.C., Vergani, F., Brogna, C., de Lucas, E.M., VázquezBarquero, A., García-Porrero, J.A., Duffau, H., 2011. Cortex-sparing fiber dissection: An improved method for the study of white matter anatomy in the human brain. J. Anat. 219, 531-541. https://doi.org/10.1111/j.1469-7580.2011.01414.x

Martino, J., Vergani, F., Robles, S.G., Duffau, H., 2010. New Insights Into the Anatomic Dissection of the Temporal Stem With Special Emphasis on the Inferior Frontooccipital Fasciculus: Implications in Surgical Approach to Left Mesiotemporal and 
Temporoinsular Structures. Oper. Neurosurg. 66, ons-4-ons-12. https://doi.org/10.1227/01.NEU.0000348564.28415.FA

Mayo, H., 1823. Anatomical and Physiological Commentaries, Number II. London: Underwood.

Monroy-Sosa, A., Jennings, J., Chakravarthi, S., Fukui, M.B., Celix, J., Kojis, N., Lindsay, M., Walia, S., Rovin, R., Kassam, A., 2019. Microsurgical Anatomy of the Vertical Rami of the Superior Longitudinal Fasciculus: An Intraparietal Sulcus Dissection Study. Oper. Neurosurg. 16, 226-238. https://doi.org/10.1093/ons/opy077

Mori, S., Kaufmann, W.E., Davatzikos, C., Stieltjes, B., Amodei, L., Fredericksen, K., Pearlson, G.D., Melhem, E.R., Solaiyappan, M., Raymond, G. V., Moser, H.W., Van Zijl, P.C.M., 2002. Imaging cortical association tracts in the human brain using diffusion-tensor-based axonal tracking. Magn. Reson. Med. 47, 215-223. https://doi.org/10.1002/mrm.10074

Mortazavi, F., Oblak, A.L., Morrison, W.Z., Schmahmann, J.D., Stanley, H.E., Wedeen, V.J., Rosene, D.L., 2018. Geometric Navigation of Axons in a Cerebral Pathway: Comparing dMRI with Tract Tracing and Immunohistochemistry. Cereb. Cortex 28, 1219-1232. https://doi.org/10.1093/cercor/bhx034

Nakajima, R., Kinoshita, M., Shinohara, H., Nakada, M., 2019. The superior longitudinal fascicle: reconsidering the fronto-parietal neural network based on anatomy and function. Brain Imaging Behav. https://doi.org/10.1007/s11682-019-00187-4

Panesar, S.S., Belo, J.T.A., Yeh, F.-C., Fernandez-Miranda, J.C., 2019. Structure, asymmetry, and connectivity of the human temporo-parietal aslant and vertical occipital fasciculi. Brain Struct. Funct. 224, 907-923. https://doi.org/10.1007/s00429-018-1812-0

Panesar, S.S., Fernandez-Miranda, J., 2019. Commentary: The Nomenclature of Human White Matter Association Pathways: Proposal for a Systematic Taxonomic Anatomical Classification. Front. Neuroanat. 13, 61. https://doi.org/10.3389/fnana.2019.00061

Panesar, S.S., Yeh, F.-C., Jacquesson, T., Hula, W., Fernandez-Miranda, J.C., 2018. A Quantitative Tractography Study Into the Connectivity, Segmentation and Laterality of the Human Inferior Longitudinal Fasciculus. Front. Neuroanat. 12, 47. https://doi.org/10.3389/fnana.2018.00047

Papagno, C., Comi, A., Riva, M., Bizzi, A., Vernice, M., Casarotti, A., Fava, E., Bello, L., 2017. Mapping the brain network of the phonological loop. Hum. Brain Mapp. 38, 3011-3024.

Petrides, M., Pandya, D.N., 1984. Projections to the frontal cortex from the posterior parietal region in the rhesus monkey. J. Comp. Neurol. 228, 105-116. https://doi.org/10.1002/cne.902280110

Rajkowska, G., Goldman-Rakic, P., 1995. Cytoarchitectonic definition of prefrontal areas in the normal human cortex: 2. Variability in locations of areas 9 and 46 and relationship to the Talairach coordinate system. Cereb. Cortex 323-337.

Reil, J.C., 1812. Die vördere commissur im groben gehirn. Arch. Für Physiol. 11, 89-100.

Reil, J.C., 1809. Die Sylvische Grube oder das Thal, das gestreifte grobe hirnganglium, dessen kapsel und die seitentheile des grobn gehirns. Arch. Für Physiol. 9, 195-208.

Rheault, F., De Benedictis, A., Daducci, A., Maffei, C., Tax, C.M.W., Romascano, D., Caverzasi, E., Morency, F.C., Corrivetti, F., Pestilli, F., Girard, G., Theaud, G., Zemmoura, I., Hau, J., Glavin, K., Jordan, K.M., Pomiecko, K., Chamberland, M., Barakovic, M., Goyette, N., Poulin, P., Chenot, Q., Panesar, S.S., Sarubbo, S., Petit, L., Descoteaux, M., 2020a. Tractostorm: The what, why, and how of tractography dissection reproducibility. Hum. Brain Mapp. 41, 1859-1874. https://doi.org/10.1002/hbm.24917 
Rheault, F., Poulin, P., Valcourt Caron, A., St-Onge, E., Descoteaux, M., 2020b. Common misconceptions, hidden biases and modern challenges of dMRI tractography. J. Neural Eng. 17, 011001. https://doi.org/10.1088/1741-2552/ab6aad

Rojkova, K., Volle, E., Urbanski, M., Humbert, F., Dell'Acqua, F., Thiebaut de Schotten, M., 2016. Atlasing the frontal lobe connections and their variability due to age and education: a spherical deconvolution tractography study. Brain Struct. Funct. 221, 1751-1766. https://doi.org/10.1007/s00429-015-1001-3

Sarubbo, S., Basso, G., Chioffi, F., Cesnik, E., Paradiso, B., Grandi, E., Fainardi, E., Tugnoli, V., Farneti, M., Granieri, E., 2013a. Technical, Anatomical, and Functional Study after Removal of a Symptomatic Cavernous Angioma Located in Deep Wernicke's Territories with Cortico-Subcortical Awake Mapping. Case Rep. Neurol. Med. 2013, 1-7. https://doi.org/10.1155/2013/835029

Sarubbo, S., De Benedictis, A., Maldonado, I.L., Basso, G., Duffau, H., 2013b. Frontal terminations for the inferior fronto-occipital fascicle: anatomical dissection, DTI study and functional considerations on a multi-component bundle. Brain Struct. Funct. 218, 21-37. https://doi.org/10.1007/s00429-011-0372-3

Sarubbo, S., De Benedictis, A., Merler, S., Mandonnet, E., Balbi, S., Granieri, E., Duffau, H., 2015. Towards a functional atlas of human white matter: Functional Atlas of White Matter. Hum. Brain Mapp. 36, 3117-3136. https://doi.org/10.1002/hbm.22832

Sarubbo, S., De Benedictis, A., Merler, S., Mandonnet, E., Barbareschi, M., Dallabona, M., Chioffi, F., Duffau, H., 2016. Structural and functional integration between dorsal and ventral language streams as revealed by blunt dissection and direct electrical stimulation: Anatomo-Functional Integration of Language. Hum. Brain Mapp. 37, 3858-3872. https://doi.org/10.1002/hbm.23281

Sarubbo, S., Petit, L., De Benedictis, A., Chioffi, F., Ptito, M., Dyrby, T.B., 2019. Uncovering the inferior fronto-occipital fascicle and its topological organization in non-human primates: the missing connection for language evolution. Brain Struct. Funct. 224, 1553-1567. https://doi.org/10.1007/s00429-019-01856-2

Sarubbo, S., Tate, M., De Benedictis, A., Merler, S., Moritz-Gasser, S., Herbet, G., Duffau, H., 2020a. Mapping critical cortical hubs and white matter pathways by direct electrical stimulation: an original functional atlas of the human brain. NeuroImage 205, 116237. https://doi.org/10.1016/j.neuroimage.2019.116237

Sarubbo, S., Tate, M., De Benedictis, A., Merler, S., Moritz-Gasser, S., Herbet, G., Duffau, H., 2020b. Mapping critical cortical hubs and white matter pathways by direct electrical stimulation: an original functional atlas of the human brain. NeuroImage 205, 116237. https://doi.org/10.1016/j.neuroimage.2019.116237

Schmahmann, J.D., Pandya, D.N., 2006. Fiber Pathways of the Brain. Oxford University Press. https://doi.org/10.1093/acprof:oso/9780195104233.001.0001

Schmahmann, J.D., Pandya, D.N., Wang, R., Dai, G., D’Arceuil, H.E., de Crespigny, A.J., Wedeen, V.J., 2007. Association fibre pathways of the brain: parallel observations from diffusion spectrum imaging and autoradiography. Brain 130, 630-653. https://doi.org/10.1093/brain/awl359

Schurr, R., Zelman, A., Mezer, A.A., 2020. Subdividing the superior longitudinal fasciculus using local quantitative MRI. NeuroImage 208, 116439. https://doi.org/10.1016/j.neuroimage.2019.116439

Spena, G., Gatignol, P., Capelle, L., Duffau, H., 2006. Superior longitudinal fasciculus subserves vestibular network in humans: NeuroReport 17, 1403-1406. https://doi.org/10.1097/01.wnr.0000223385.49919.61

Swanson, L.W., 2015. Neuroanatomical terminology: a lexicon of classical origins and historical foundations. Oxford University Press, Oxford ; New York. 
Thiebaut de Schotten, M., 2005. Direct Evidence for a Parietal-Frontal Pathway Subserving Spatial Awareness in Humans. Science 309, 2226-2228. https://doi.org/10.1126/science.1116251

Thiebaut de Schotten, M., Dell'Acqua, F., Forkel, S.J., Simmons, A., Vergani, F., Murphy, D.G.M., Catani, M., 2011a. A lateralized brain network for visuospatial attention. Nat. Neurosci. 14, 1245-1246. https://doi.org/10.1038/nn.2905

Thiebaut de Schotten, M., Dell'Acqua, F., Valabregue, R., Catani, M., 2012. Monkey to human comparative anatomy of the frontal lobe association tracts. Cortex 48, 82-96. https://doi.org/10.1016/j.cortex.2011.10.001

Thiebaut de Schotten, M., ffytche, D.H., Bizzi, A., Dell'Acqua, F., Allin, M., Walshe, M., Murray, R., Williams, S.C., Murphy, D.G.M., Catani, M., 2011b. Atlasing location, asymmetry and inter-subject variability of white matter tracts in the human brain with MR diffusion tractography. NeuroImage 54, 49-59. https://doi.org/10.1016/j.neuroimage.2010.07.055

Tournier, J.-D., Calamante, F., Connelly, A., 2007. Robust determination of the fibre orientation distribution in diffusion MRI: Non-negativity constrained super-resolved spherical deconvolution. NeuroImage 35, 1459-1472. https://doi.org/10.1016/j.neuroimage.2007.02.016

Türe, U., Yaşargil, M.G., Friedman, A.H., Al-Mefty, O., 2000. Fiber dissection technique: Lateral aspect of the brain. Neurosurgery 47, 417-427. https://doi.org/10.1097/00006123-200008000-00028

Tzourio, N., Petit, L., Mellet, E., Orssaud, C., Crivello, F., Benali, K., Salamon, G., Mazoyer, B., 1997. Use of anatomical parcellation to catalog and study structure-function relationships in the human brain. Hum. Brain Mapp. 5.

Umarova, R.M., Saur, D., Schnell, S., Kaller, C.P., Vry, M.-S., Glauche, V., Rijntjes, M., Hennig, J., Kiselev, V., Weiller, C., 2010. Structural Connectivity for Visuospatial Attention: Significance of Ventral Pathways. Cereb. Cortex 20, 121-129. https://doi.org/10.1093/cercor/bhp086

Vassal, F., Schneider, F., Boutet, C., Jean, B., Sontheimer, A., Lemaire, J.-J., 2016. Combined DTI Tractography and Functional MRI Study of the Language Connectome in Healthy Volunteers: Extensive Mapping of White Matter Fascicles and Cortical Activations. PLOS ONE 11, e0152614. https://doi.org/10.1371/journal.pone.0152614

von Monakow, C., 1897. Gehirnpathologie : Allgemeine Einleitung, Localisation, Gehirnblutungen, Verstopfung der Hirnarterien.

Wakana, S., Caprihan, A., Panzenboeck, M.M., Fallon, J.H., Perry, M., Gollub, R.L., Hua, K., Zhang, J., Jiang, H., Dubey, P., Blitz, A., van Zijl, P., Mori, S., 2007. Reproducibility of quantitative tractography methods applied to cerebral white matter. NeuroImage 36, 630-644. https://doi.org/10.1016/j.neuroimage.2007.02.049

Wang, X., Pathak, S., Stefaneanu, L., Yeh, F.-C., Li, S., Fernandez-Miranda, J.C., 2016. Subcomponents and connectivity of the superior longitudinal fasciculus in the human brain. Brain Struct. Funct. 221, 2075-2092. https://doi.org/10.1007/s00429-015-10285

Wu, Y., Sun, D., Wang, Yong, Wang, Yibao, Ou, S., 2016a. Segmentation of the Cingulum Bundle in the Human Brain: A New Perspective Based on DSI Tractography and Fiber Dissection Study. Front. Neuroanat. 10. https://doi.org/10.3389/fnana.2016.00084

Wu, Y., Sun, D., Wang, Yong, Wang, Yunjie, Wang, Yibao, 2016b. Tracing short connections of the temporo-parieto-occipital region in the human brain using diffusion spectrum imaging and fiber dissection. Brain Res. 1646, 152-159. https://doi.org/10.1016/j.brainres.2016.05.046 
Yagmurlu, K., Middlebrooks, E.H., Tanriover, N., Rhoton, A.L., 2016. Fiber tracts of the dorsal language stream in the human brain. J. Neurosurg. 124, 1396-1405. https://doi.org/10.3171/2015.5.JNS15455

Zacà, D., Corsini, F., Rozzanigo, U., Dallabona, M., Avesani, P., Annicchiarico, L., Zigiotto, L., Faraca, G., Chioffi, F., Jovicich, J., Sarubbo, S., 2018. Whole-Brain Network Connectivity Underlying the Human Speech Articulation as Emerged Integrating Direct Electric Stimulation, Resting State fMRI and Tractography. Front. Hum. Neurosci. 12, 405. https://doi.org/10.3389/fnhum.2018.00405

Zemmoura, I., Blanchard, E., Raynal, P.-I., Rousselot-Denis, C., Destrieux, C., Velut, S., 2016. How Klingler's dissection permits exploration of brain structural connectivity? An electron microscopy study of human white matter. Brain Struct. Funct. 221, 24772486. https://doi.org/10.1007/s00429-015-1050-7

Zemmoura, I., Serres, B., Andersson, F., Barantin, L., Tauber, C., Filipiak, I., Cottier, J.P., Venturini, G., Destrieux, C., 2014. FIBRASCAN: A novel method for 3D white matter tract reconstruction in MR space from cadaveric dissection. NeuroImage 103, 106-118. https://doi.org/10.1016/j.neuroimage.2014.09.016

Zhang, Y., Zhang, J., Oishi, K., Faria, A.V., Jiang, H., Li, X., Akhter, K., Rosa-Neto, P., Pike, G.B., Evans, A., Toga, A.W., Woods, R., Mazziotta, J.C., Miller, M.I., van Zijl, P.C.M., Mori, S., 2010. Atlas-guided tract reconstruction for automated and comprehensive examination of the white matter anatomy. NeuroImage 52, 1289-1301. https://doi.org/10.1016/j.neuroimage.2010.05.049 


\section{Figure legends}

Figure 1. Pioneering illustrations of the SLS with originally translated legends. The original label names have been kept and placed out of the image while pointing to their exact original position.

A. Adapted from the Plate XII in Reil (1812). The letter $\mathbf{f}$ indicated the "intermediate layer of medullary fasciculi which seem interposed between the convolutions and nucleus of the cerebrum" (from the translation of Mayo (1823)). B. Adapted from the Plate IX in Burdach (1822), the letter $\mathbf{f}$ indicated "the lateral covering white matter mass, particularly the arcuate fasciculus." The letter $\mathbf{k}$ designated "the edge formed by the arcuate fasciculus that extends to the frontal lobe on the lateral side of the brain." C. Adapted from Plate III in Mayo (1827). The letter $\mathbf{c}$ indicated the "fibers of a white substance, or plates resoluble into fibers, which connect the grey matter of remote convolutions of the same hemisphere." D. Adapted from Tabula $\mathrm{X}$ in Arnold (1838). The letter $\mathbf{k}$ designated the arcuate fasciculus, which was erroneously considered as having its origin from the corpus callosum, then was described as surrounding the corona radiata before to project to the temporal lobe. E. Adapted from figure 19 in Meynert (1884). The label f.arc indicated the arcuate fasciculus defined in the text as "In the parietal region, underneath the operculum, the fasciculus arcuatus is most strongly developed. Anteriorly its superficial strata are continued into the convolution of the operculum. In bending downward toward the temporal lobe, its superficial bundles can be seen entering the superior temporal convolution, the parallel fissure (author note, the superior temporal sulcus), and the second temporal convolution. The deeper bundles of the fasciculus arcuatus spread to the more distant convolutions of the entire convexity."

Figure 2. Illustrations of the Dejerines definition of the "faisceau arqué de Burdach ou faisceau longitudinal supérieur » adapted and translated from the figures 376 (top) and 377 (bottom) on pages 755 and 757 of Dejerine J, Dejerine-Klumpke A (1895), respectively.

Translation of the original figure 376 (top): External surface of the left hemisphere. The insula's convolutions, the Sylvian operculum, and the deep side of the first temporal convolution have been removed to show the fibers of the external capsule, the uncinate fascicle, and the arcuate or superior longitudinal fascicle (semi-schematic).

Translation of the legend of the original figure 377 (bottom): External surface of the left hemisphere. The edges of the Sylvius fissure have been pushed aside to show the insular and 
the retro-insular region. The uncinate, arcuate, or superior longitudinal and vertical occipital fascicles are seen through.

Each original label was replaced in its original location, and for easier reading, the gyri were marked in red, the sulci in green, and the bundles in blue.

Current translation of the different labels : Arc, arcuate or superior longitudinal fascicle; Ce, external capsule; F2, F3, middle and inferior frontal gyrus; $\mathbf{F 3}(\mathbf{C})$, course of the inferior frontal gyrus; f2, inferior frontal sulcus; f3, orbital or H-shaped sulcus; f4, olfactory sulcus; Fa, precentral gyrus; fa(ip), U-shaped fibers of the intraparietal sulcus; fa(pri), U-shaped fibers of the inferior part of the precental sulcus; Fu, uncinate fascicle; Ia, Ip, short and long insular gyri; io, intraoccipital sulcus; ip, intraparietal sulcus; ma, mp, ms, anterior, posterior and superior parts of the marginal or circular sulcus; O1, O2, O3, superior, middle and inferior occipital gyri; oa, anterior occipital sulcus; $\mathbf{0 F 1 , ~ o F 2 , ~ o F 3 , ~ o r b i t a l ~ p a r t ~ o f ~ s u p e r i o r , ~ m i d d l e ~ a n d ~}$ inferior frontal gyri; oF1(Gr), rectus gyrus; OpR, Rolandic opercule; OpP2, parietal opercule; Ov, vertical occipital fascicle; P1, P2, superior and inferior parietal gyri; Pa, postcentral gyrus; Pc, angular gyrus (pli courbe); po, parieto-occipital fissure; por, postcentral sulcus; pri, inferior precentral sulcus, prs, superior precentral gyrus; $\mathbf{R}$, Rolando fissure (central sulcus); $\mathbf{S}(\mathbf{a}), \mathbf{S}(\mathbf{v})$, horizontal and vertical branches of the Silvius fissure; T1, T2, superior and middle temporal gyri; t1, superior temporal sulcus; t1', vertical branches of the superior temporal sulcus; t2, inferior temporal sulcus; Tp, Heschl gyrus; VS, Sylvian fissure.

Figure 3. Illustration of the superior frontal fasciculus adapted and translated from Tabulae 5 and 6 in Ludwig E, Klingler J (1956) Atlas cerebri humani. The original label names have been kept and placed while pointing to their exact original positions. Tabula 5 (top): 1. Insula, 2. Olfactory tract, 3. Uncinate fasciculus, 4. Optic chiasma, 5. Inferior fronto-occipital fasciculus, 6. Superior frontal fasciculus. Tabula 6 (bottom): 1. Superior frontal fasciculus; 2 . The white matter of the insula, 3. Uncinate fasciculus, 4. Fasciculus of the insula; 5. Inferior frontooccipital fasciculus, 6. Superior frontal fasciculus.

Figure 4. Schematic representation and wiring diagram of the AF (in red) intended as a whole and direct connection linking the frontal to the temporal lobe. The width of each line is proportional to the number of studies supporting a specific proposal. The descriptions provided by the literature, referenced for each ROI, have been re-defined with the adoption of a gyral terminology, in particular: dorsolateral frontal cortex $=$ MFG; ventral premotor cortex $=$ vPrCG; frontal operculum = vPrCG, IFGop, IFGtri. 
Figure 5. Schematic representation and wiring diagrams of the a. AF and the b. white matter fibers of the perisylvian region according to the tripartite subdivision, comprising the anterior segment (in green), the posterior segment (in yellow), and the direct component or 'classical AF' (in red). The width of each line is proportional to the number of studies supporting a specific proposal. The descriptions provided by the literature, referenced for each ROI, have been re-defined with the adoption of a gyral terminology, in particular: ventrolateral frontal cortex $=\mathrm{IFG}, \mathrm{MFG} ;$ ventral premotor cortex $=\mathrm{vPrCG} ;$ auditory cortex $=\mathrm{STG}$.

Figure 6. Schematic representation and wiring diagram of the AF according to the STG or inner/ventral pathway (in light red) and MTG or outer/dorsal pathway (in dark red) partitioning. The width of each line is proportional to the number of studies supporting a specific proposal. The descriptions provided by the literature, referenced for each ROI, have been re-defined with the adoption of a gyral terminology, in particular: ventral premotor cortex $=\mathrm{vPrCG}$.

Figure 7. Schematic representation and wiring diagram of the SLF according to the SLF I-III segmentation. The width of each line is proportional to the number of studies supporting a specific proposal. The descriptions provided by the literature, referenced for each ROI, have been re-defined with the adoption of a gyral terminology, in particular: dorsal premotor area $=$ SFG; dorsolateral prefrontal cortex $=\mathrm{MFG}$

Figure 8. Schematic representation and wiring diagram of the SLF according to its subdivision into frontoparietal (in green) and parietotemporal components (in yellow). The width of each line is proportional to the number of studies supporting a specific proposal. The descriptions provided by the literature, referenced for each ROI, have been re-defined with the adoption of a gyral terminology, in particular: frontal operculum = vPrCG, IFGop, IFGtri; ventral premotor cortex $=\mathrm{vPrCG}$.

Figure 9. Illustration of the proposal for a medio-lateral palisading arrangement of the SLS. A: anterior, P: posterior. 
Figure 1

A. Reil (1809)

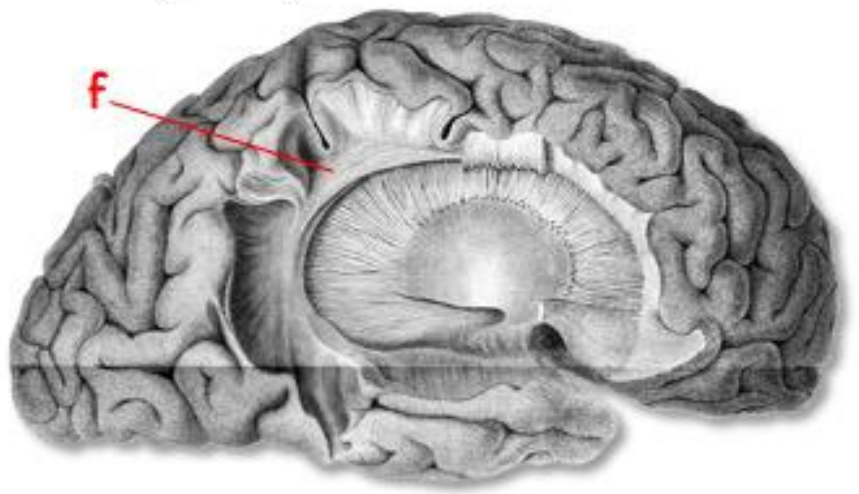

B. Burdach (1822)

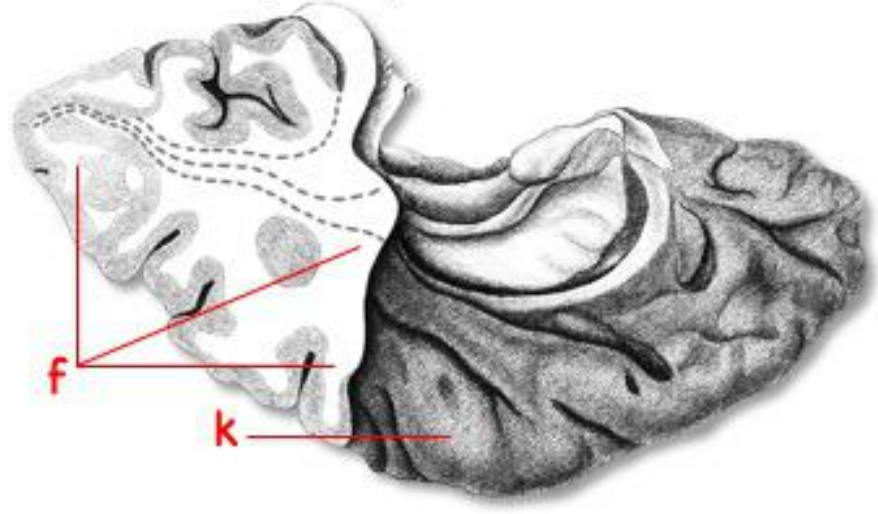

D. Arnold (1838)

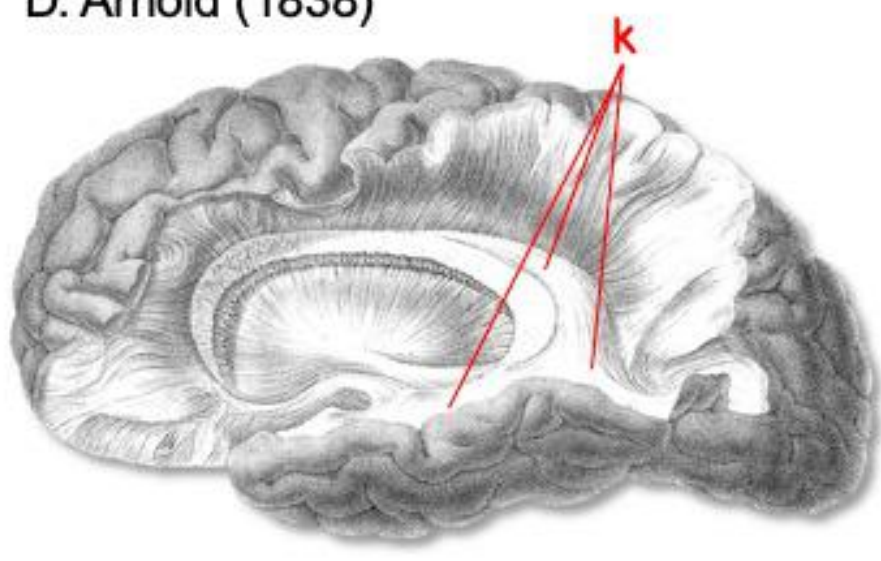

C. Mayo (1827)

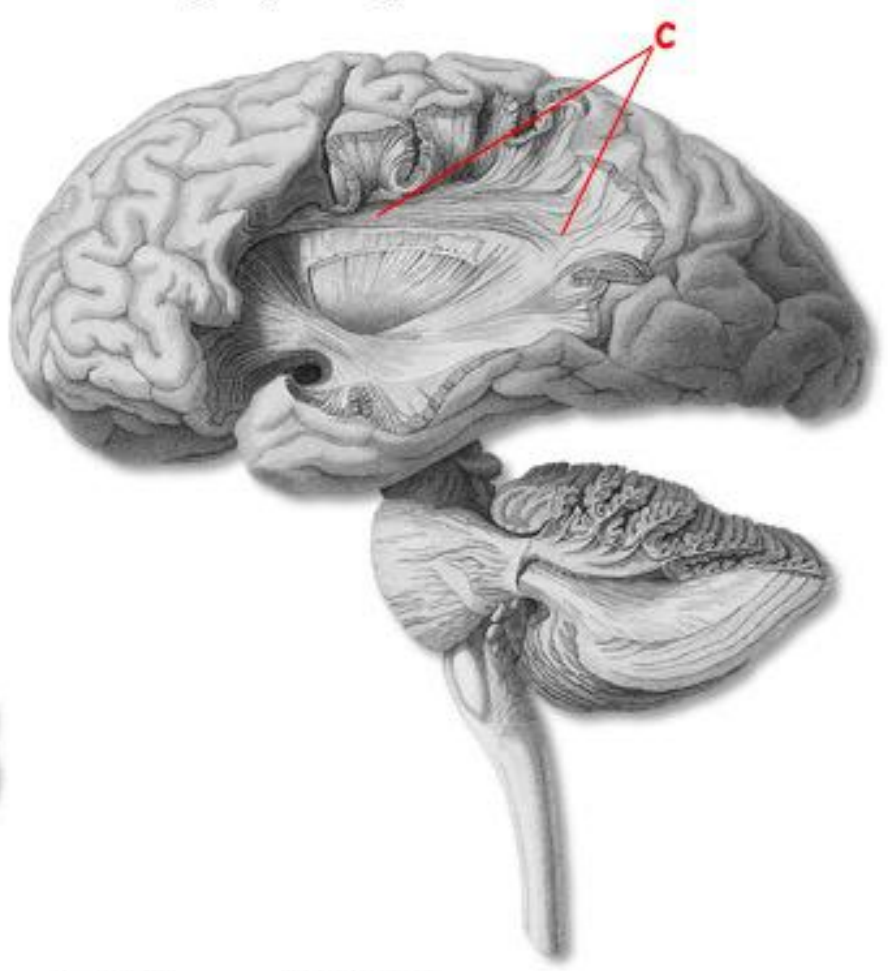

E. Meynert (1884)

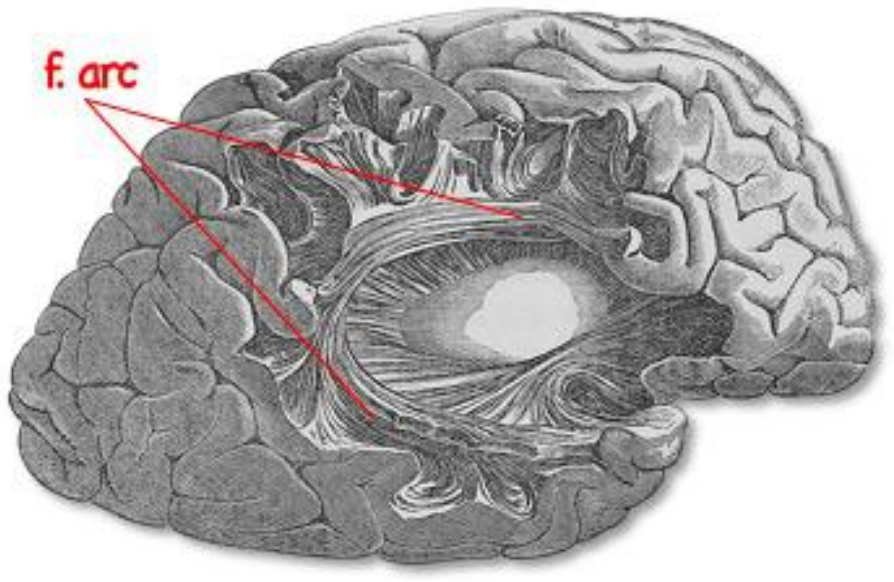




\section{Figure 2}
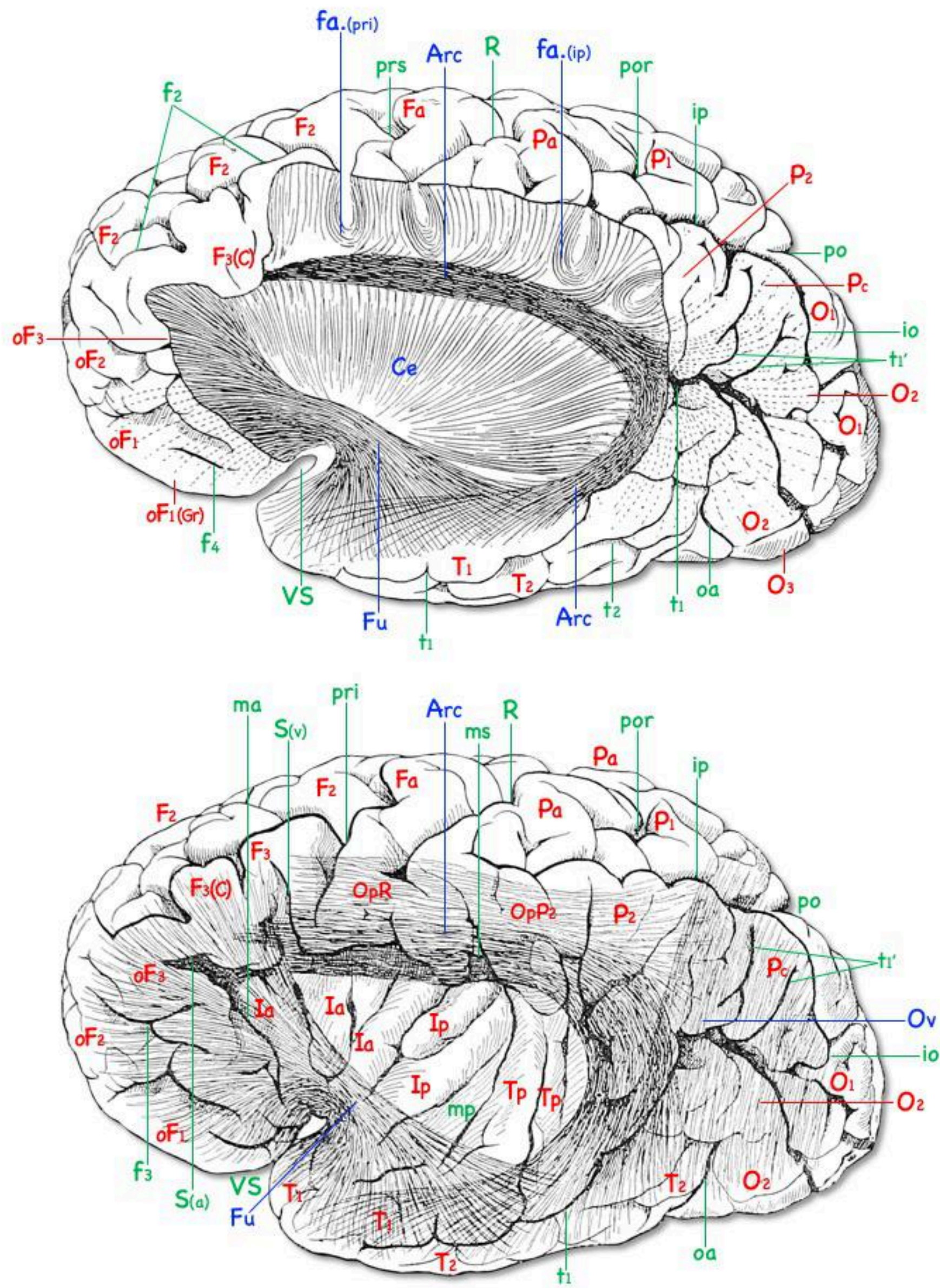
Figure 3
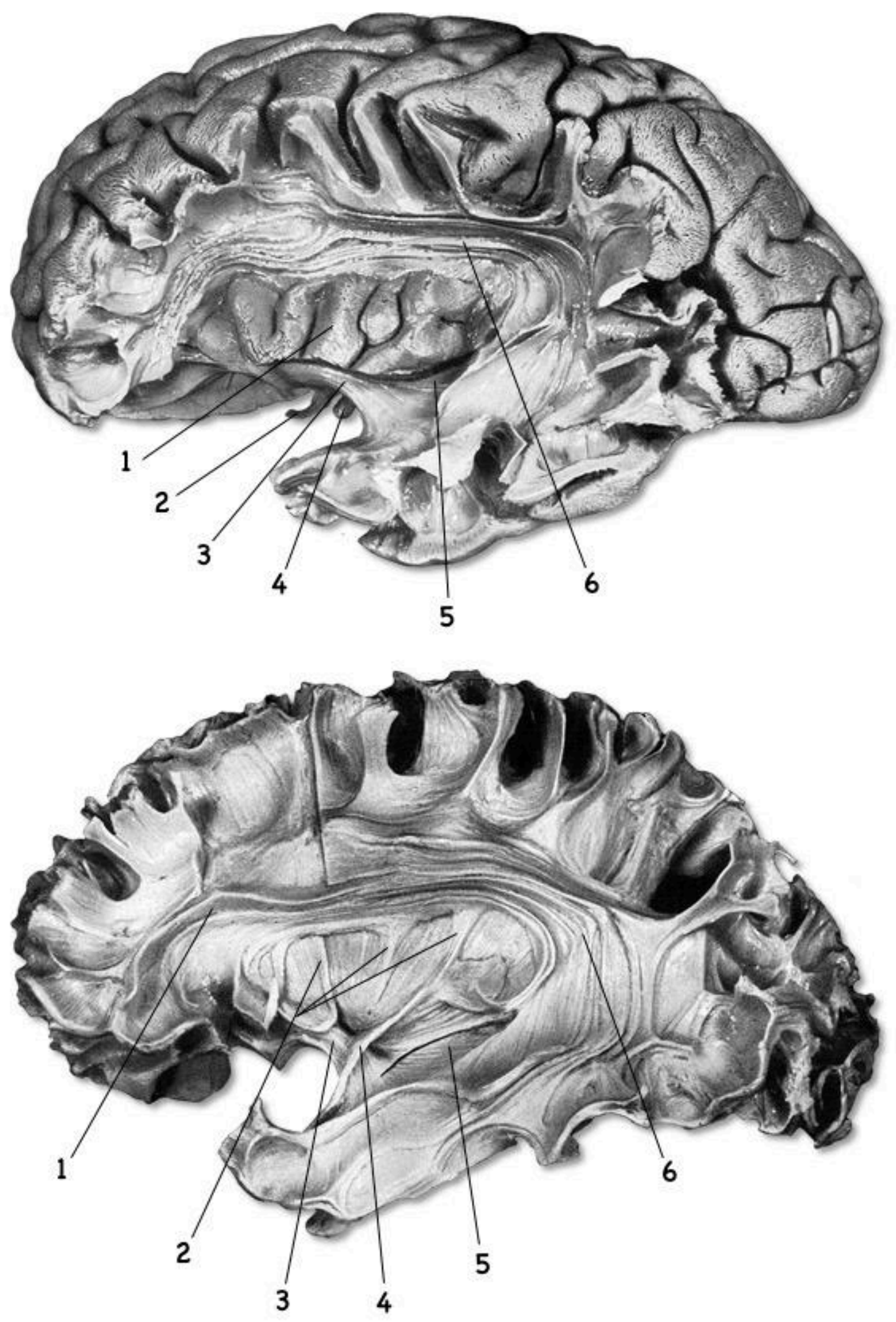


\section{Figure 4}

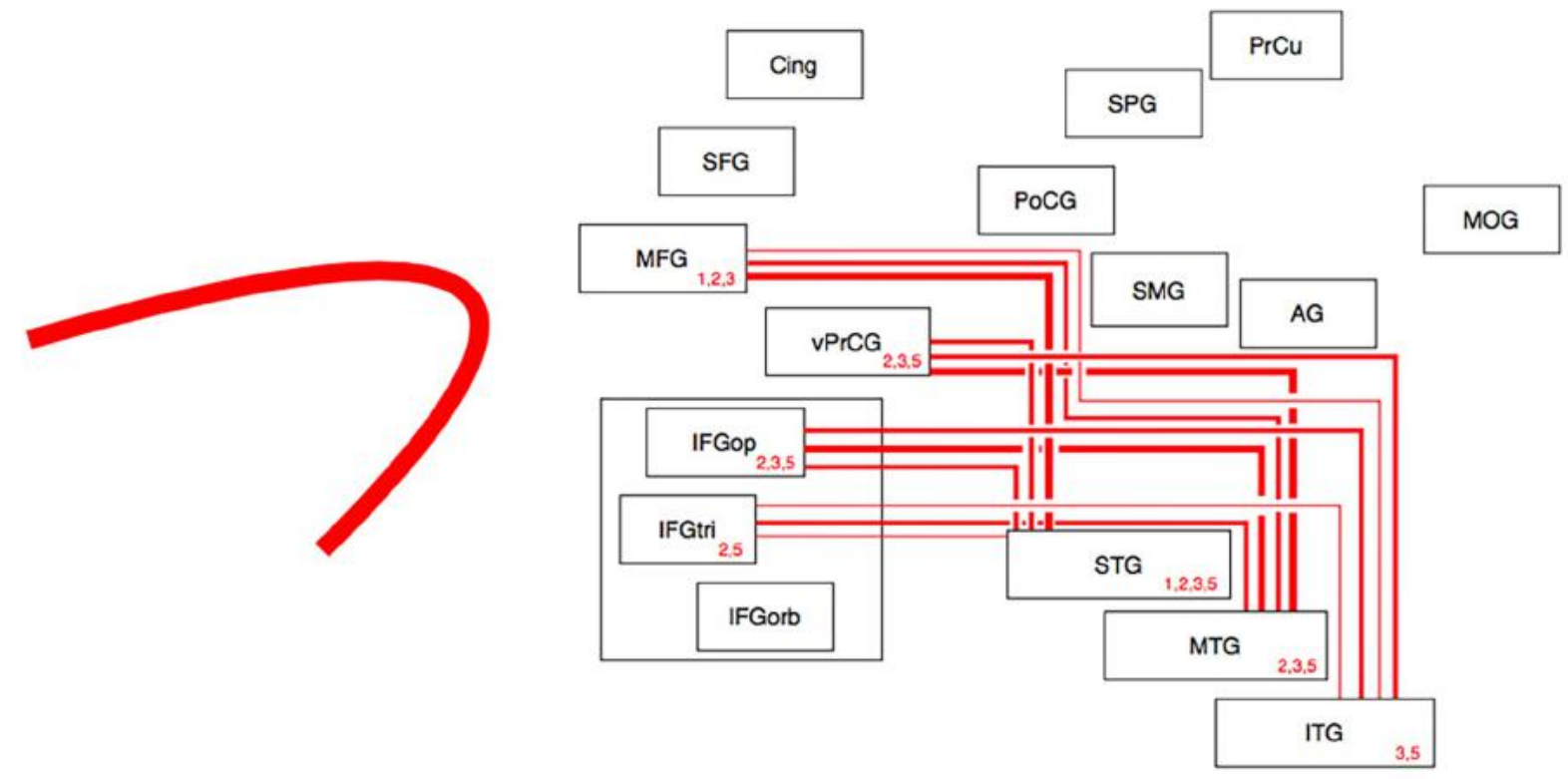

- AF (Frey et al., 20081; Vassal et al., 2016 2 ; Sarubbo et al., 2016 ${ }^{3}$ ) or arcuate segment of the SLF (Fernández-Miranda et al., 2008 ${ }^{4}$ ) or SLF temporofrontal segment (Martino et al., 20115) 


\section{Figure 5}

a.
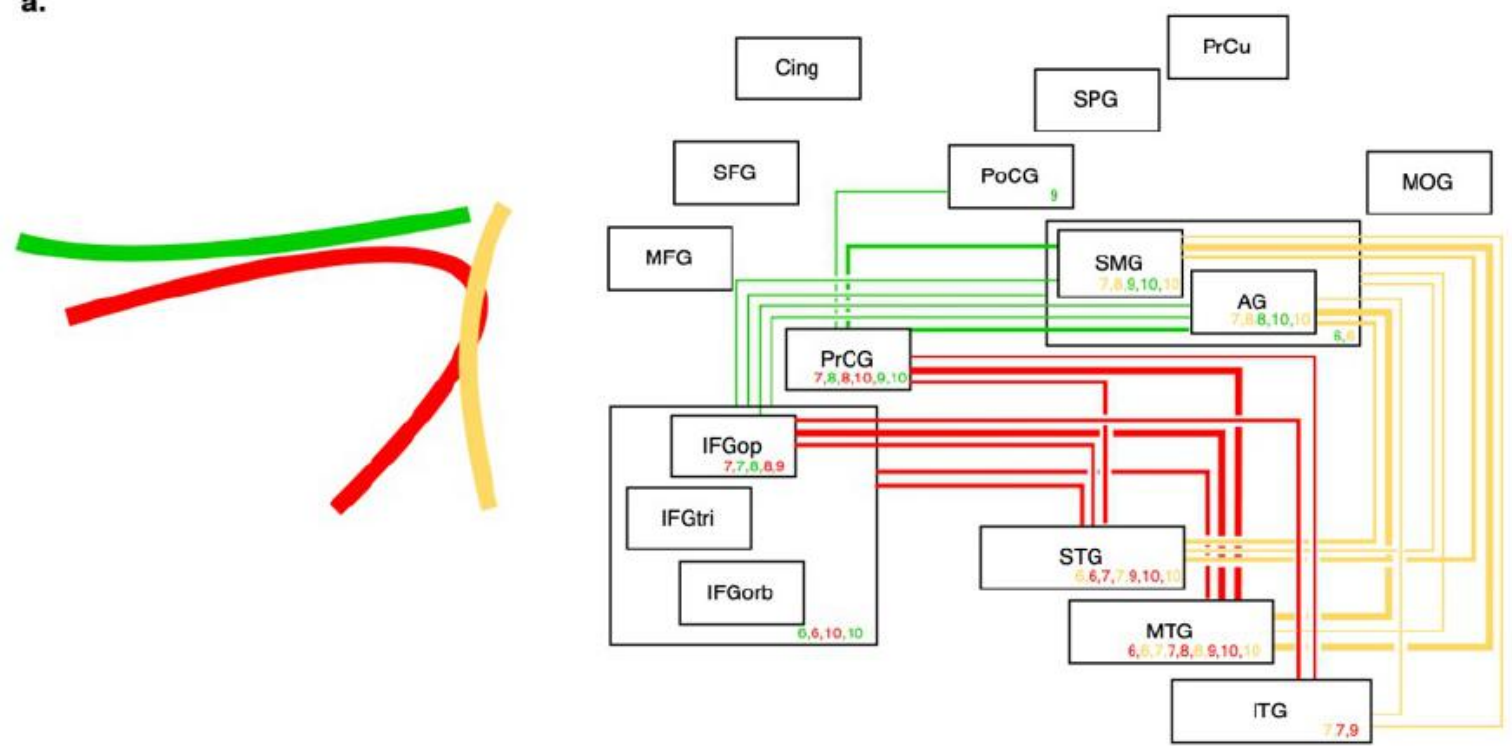

- Long segment or direct component of the AF (Catani et al., 20056 ; Barrick et al., 2006 ${ }^{7}$; Lawes et al., 2008 ${ }^{8}$; Rojkova et al., $2016^{9}$; Forkel et al., 2020 ${ }^{10}$ )

- Anterior segment of the AF (Catani et al., 20056; Lawes et al., 2008 ${ }^{8}$; Rojkova et al., 2016 ${ }^{9}$; Forkel et al., 202010)

- Postenor segment of the AF (Catani et al., 20056; Barrick et al., 20067; Lawes et al. 20088 ; Forkel et al. 202010)

b.
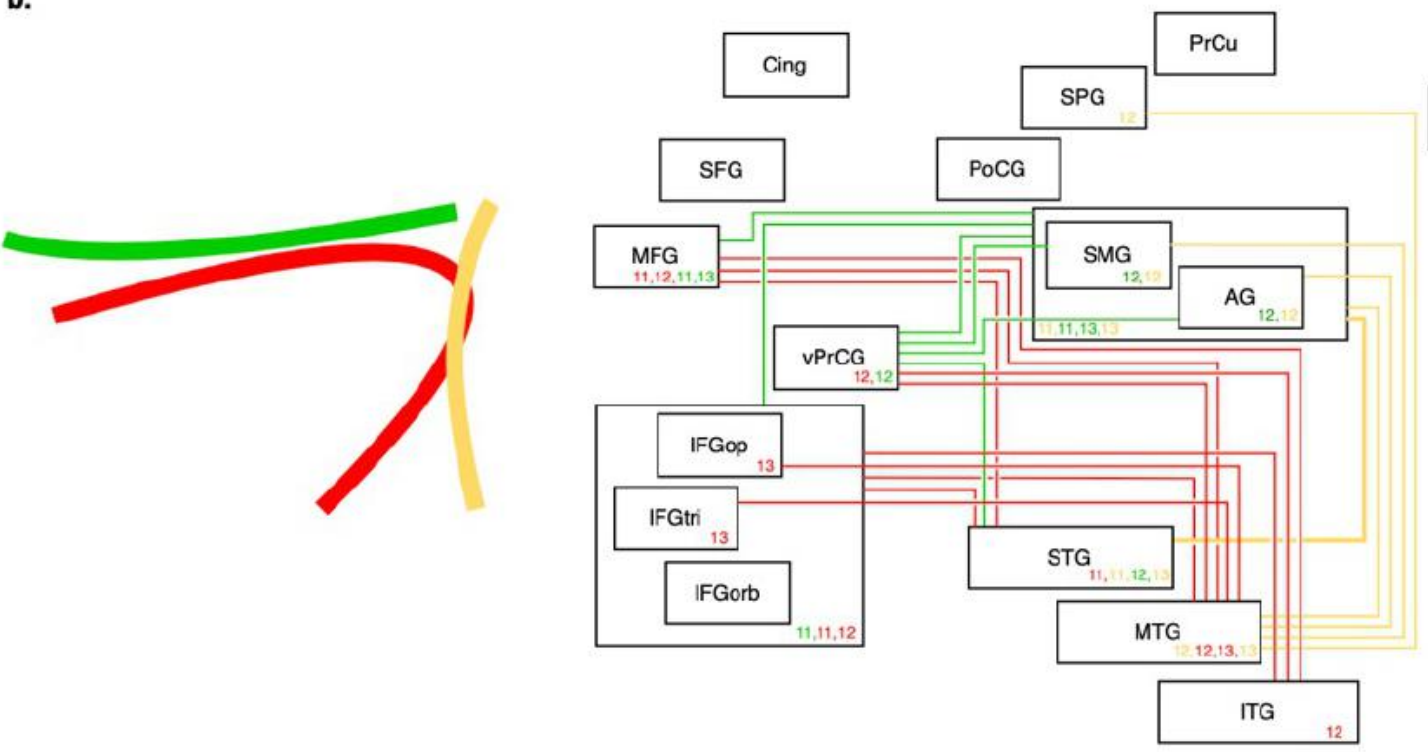

- Direct component or classical AF (Gharabaghi et al., 200911; Martino et al., 201312; Zemmoura et al., 201413)

- Anterior segment (Gharabaghi et al., 200911; Martino et al., 201312; Zemmoura et al., 201413)

(Gharabaghi et al., 200911; Martino et al., 201312; Zemmoura et al., 201413) 


\section{Figure 6}

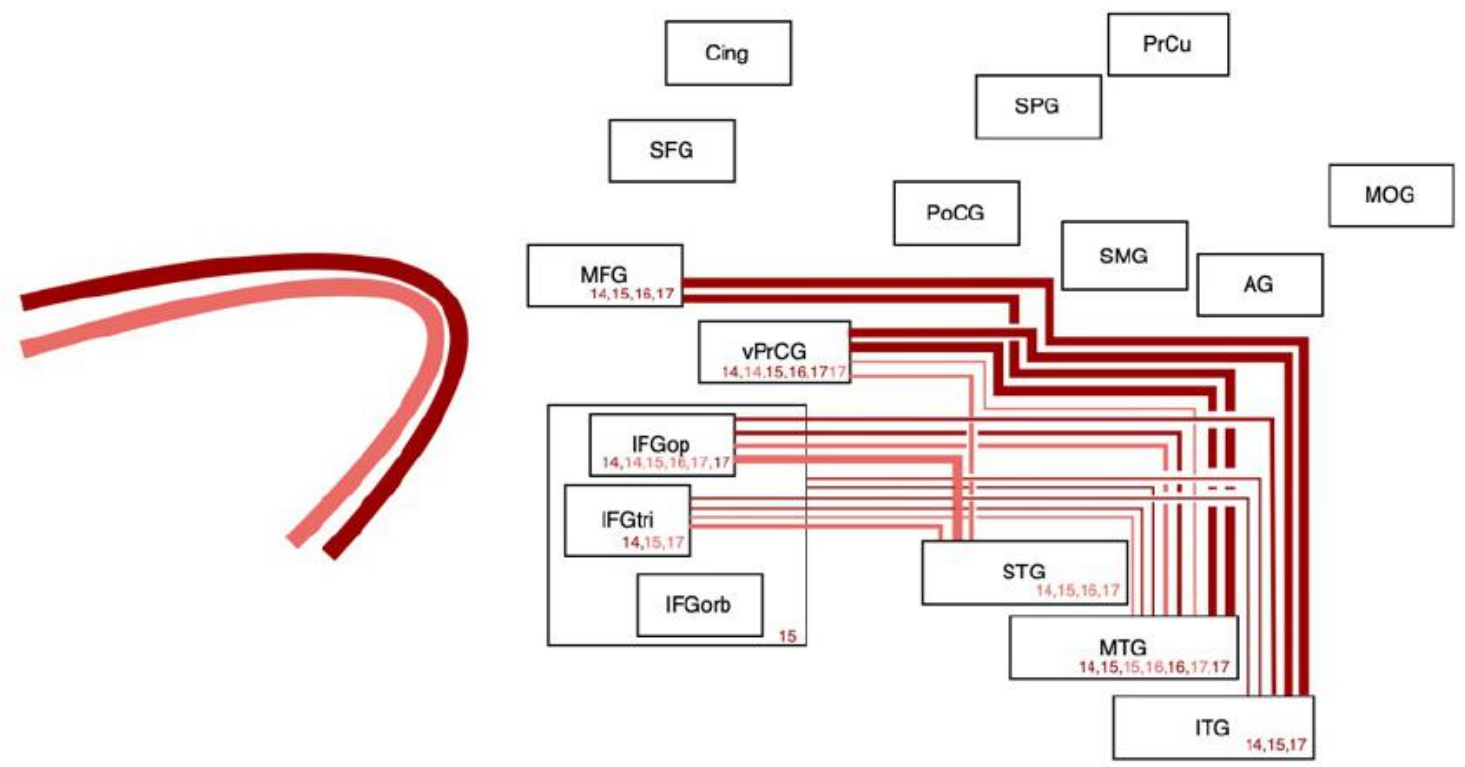

- STG pathway (Glasser and Rilling, 2008 ${ }^{14}$; Thiebaut de Schotten et al., 2012 ${ }^{15}$ ) or inner/ventral pathway (Fernández-Miranda et al., 2015 ${ }^{16}$; Yagmurlu et al., 2016 ${ }^{17}$ )

- MTG pathway (Glasser and Rilling, $2008^{14}$; Thiebaut de Schotten et al., 2012 ${ }^{15}$ ) or outer/dorsal pathway (Fernández-Miranda et al., 2015 ${ }^{16}$; Yagmurlu et al., 2016 ${ }^{17}$ ) 


\section{Figure 7}

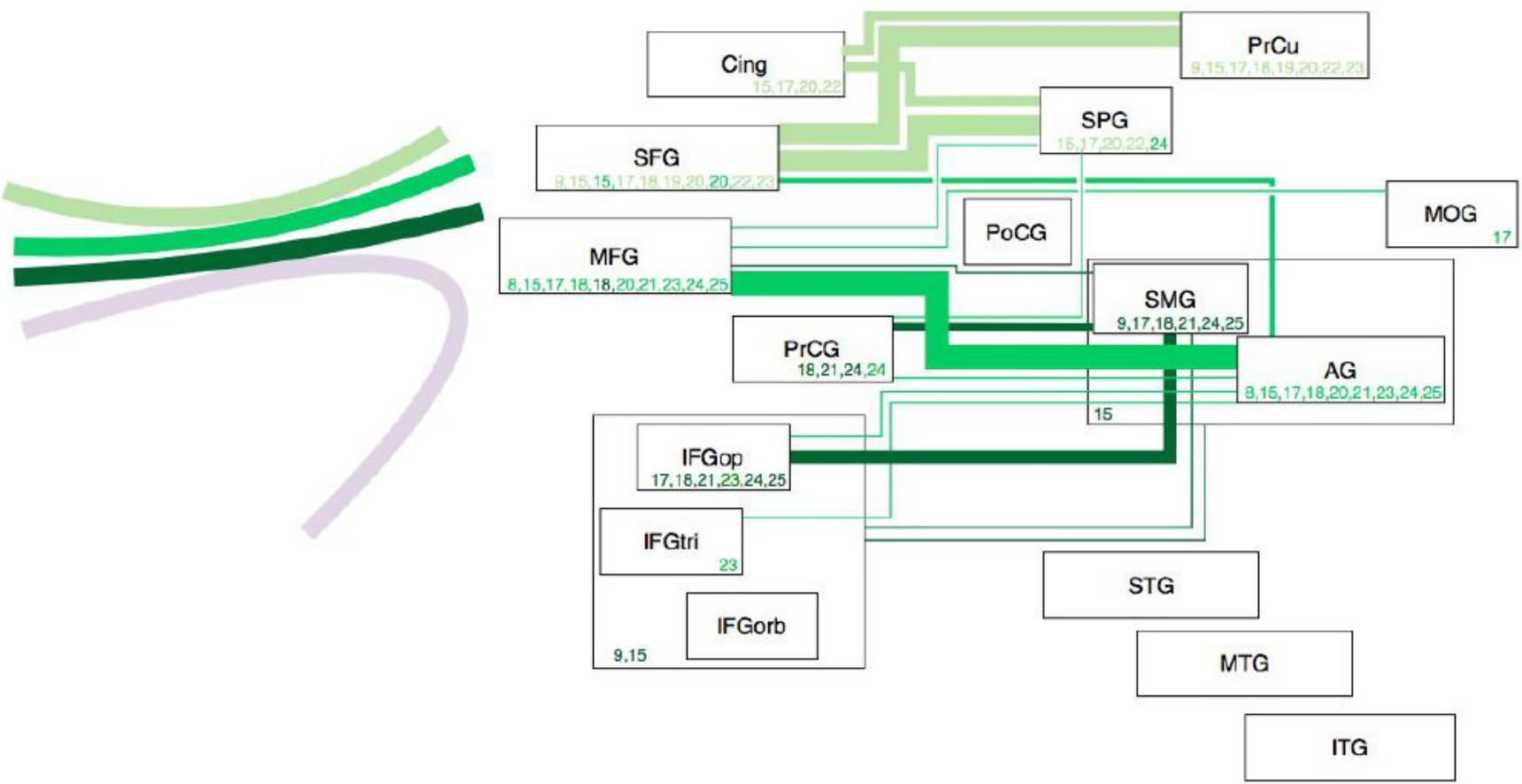

(Makris et al., 2005 ${ }^{18}$; Jang and Hong, 201219; Thiebaut de Schotten et al., 201120; Thiebaut de Schotten et al., 2012 ${ }^{15}$;

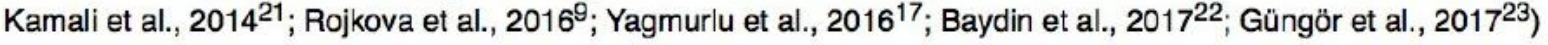

SLF II (Makris et al., 2005 ${ }^{18}$; Thiebaut de Schotten et al., 201120; Thiebaut de Schotten et al., 201215; Kamali et al., 2014 ${ }^{21}$; Rojkova et al., 20169; Wang et al., 2016 24; Yagmurlu et al., 2016 ${ }^{17}$; Baydin et al., 2017²2; Güngör et al., 201723;

Monroy-Sosa et al., 201925)

- SLF III (Makris et al., 200518; Thiebaut de Schotten et al., 201215; Kamali et al., 201421; Rojkova et al., 20169; Wang et al., 201624; Yagmurlu et al., 2016 ${ }^{17}$; Baydin et al., 201722; Güngör et al., 201723; Monroy-Sosa et al., 201925) 


\section{Figure 8}

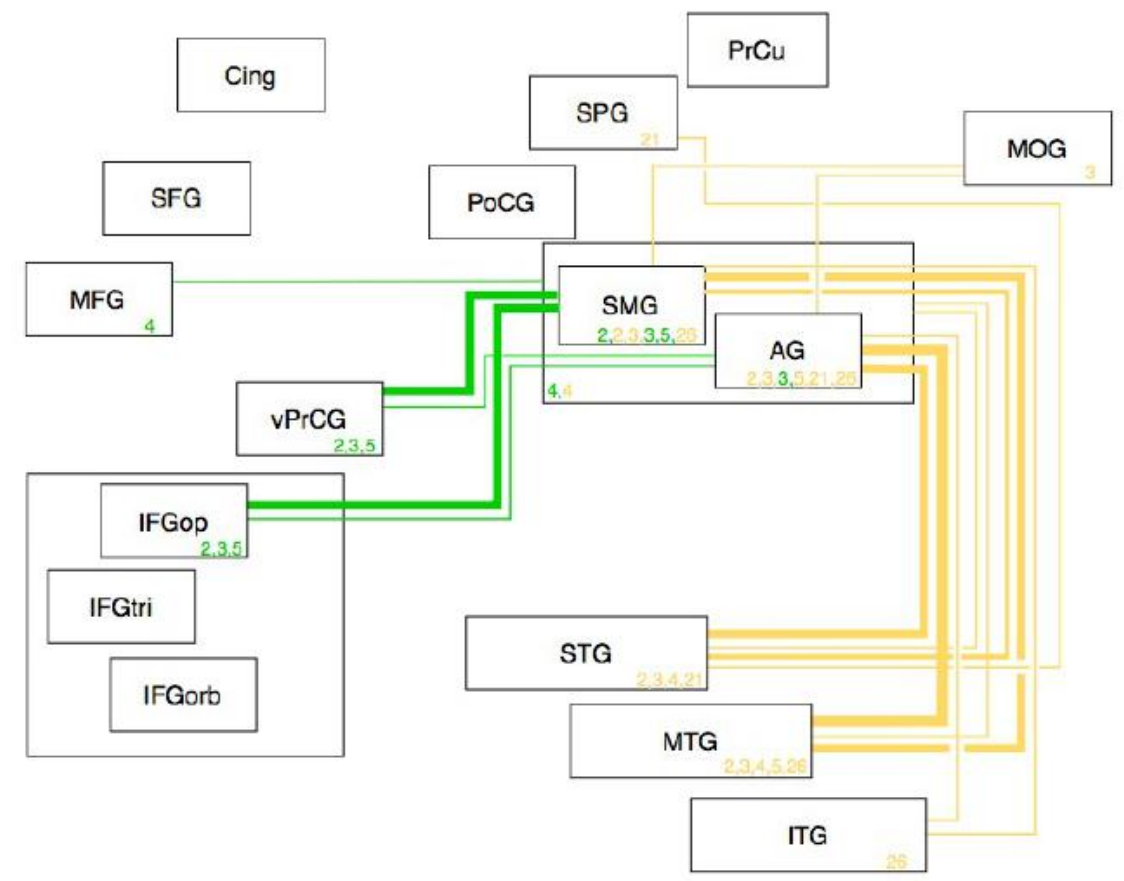

- Horizontal segment of the SLF (Fernàndez-Miranda et al., 20084; Martino et al., 20115) or anterior SLF (Sarubbo et al., 2016 ${ }^{3}$ ) or frontoparietal component of the SLF (Vassal et al., 2016 ${ }^{2}$ )

( Vertical segment of the SLF (Fernández-Miranda et al., 20084; Martino et al., 20115) or et al., $2016^{3}$ ) or parietotemporal component of the SLF (Kamali et al., 2014 $4^{21}$ ) or 


\section{Figure 9}

ㅁ.

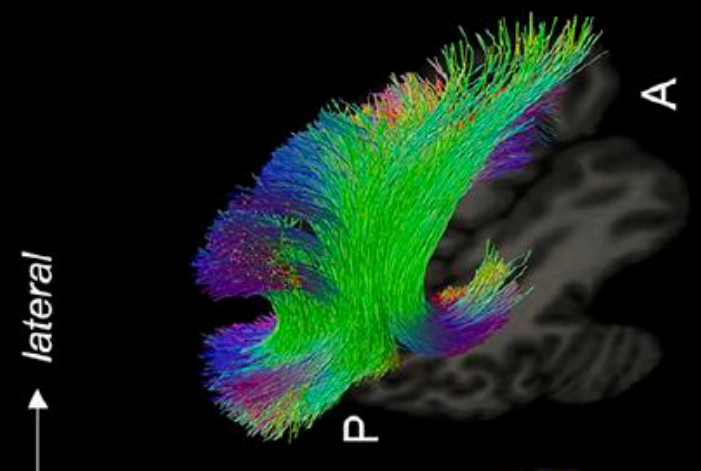

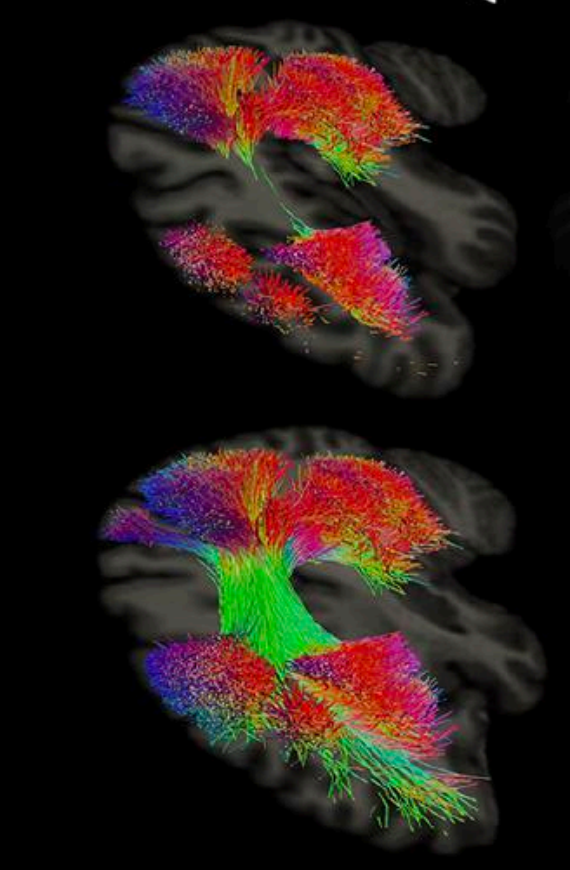
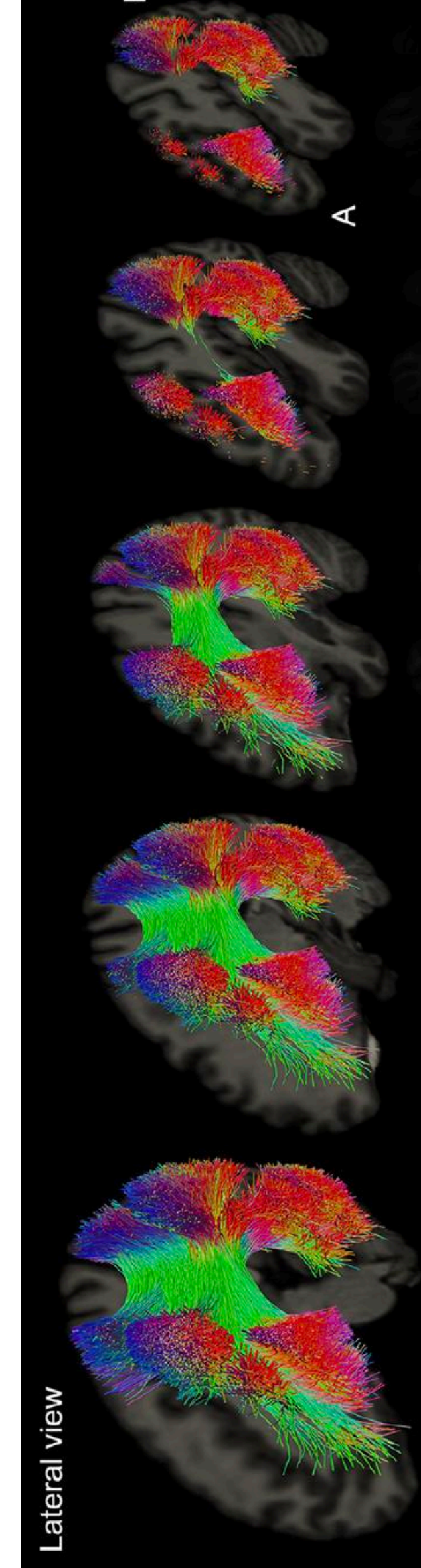

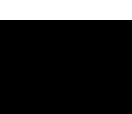

